FOLIA

Amazónica

Revista del Instituto de Investigaciones

de la Amazonía Peruana

\title{
DIVERSIDAD DE AVES EN EL INTERFLUVIO NAPO-PUTUMAYO-AMAZONAS, AL NORTE DE LA AMAZONÍA PERUANA
}

\author{
Natalia C. ANGULO-PEREZ1* ${ }^{*}$ José A. ARMAS-SILVA ${ }^{1}$, Pedro E. PÉREZ-PEÑA² \\ 1 Acciones para una Vida Ecosostenible (ONG AVE), Iquitos, Perú. \\ 2 Instituto de Investigaciones de la Amazonía Peruana (IIAP). \\ *Correo electrónico: nataliaangulo2797@gmail.com
}

\section{RESUMEN}

El estudio de los hábitats de la avifauna es fundamental para comprender los patrones de abundancia, diversidad y distribución de las especies. Por ello, se revisó bibliografía y bases de datos generadas en estudios donde se usaron transectos y redes de neblina para conocer la riqueza ornitológica en el interfluvio Napo-Putumayo-Amazonas, su composición por tipos de hábitats, especies amenazadas y principales amenazas. Se analizó la riqueza observada y esperada, así como la similitud entre los hábitats inundables (varillal pantanoso y aguajal mixto) y de tierra firme (colina baja, terraza alta y terraza media). Se compiló el registro de 558 especies, que representan más del $50 \%$ de las especies estimadas para Loreto, siendo los bosques de colina baja los que presentan la mayor riqueza de especies. La composición de especies varía por tipo de hábitat inundable y de tierra firme, pero comparten especies, siendo la terraza media la que alberga especies de bosque inundable y tierra firme. Las familias Thamnophilidae, Tyrannidae y Furnariidae son las que están mejor representadas en todos los hábitats. La avifauna es muy diversa y alberga especies únicas, amenazadas y potenciales para el aviturismo. Lamentablemente, la zona también tiene amenazas que ponen en peligro la supervivencia de las aves, como la pérdida de hábitats por la deforestación, por la agricultura migratoria, cultivo de Erythroxylum coca y/o industria maderera, tráfico de especies y sobrecaza, 
por lo que es importante implementar planes de manejo de especies, planes de conservación de hábitats prioritarios o planes turísticos que garanticen la conservación de la avifauna.

PALABRAS CLAVE: amenazas, avifauna, conservación, hábitats, interfluvio.

\title{
AVIAN DIVERSITY IN THE NAPO-PUTUMAYO-AMAZONAS INTERFLUVIUM, NORTHERN PERUVIAN AMAZON
}

\begin{abstract}
The study of avian habitats is essential to understanding species abundance, diversity and distribution. For this reason, a survey of transects and mist net studies were reviewed to ascertain the ornithological richness in the NapoPutumayo-Amazonas interfluvium, including its composition by habitat types, threatened species and main threats. Analyses conducted included a comparison of observed and expected diversity, as well as the similarities between flooded habitats (swampy varillal and mixed palm swamp) and terra firme (low hill, high terrace and middle terrace). With over 558 species registered, more than $50 \%$ of the estimated species for Loreto were represented, with the low hill forest containing the highest species richness. The composition of species varies by type of floodplain and terra firme habitat, but both habitats / ecosystems share species, with the middle terrace being home to species found in the flooded forest and terra firme. The families Thamnophilidae, Tyrannidae and Furnariidae are the best represented in all habitats. The avifauna is very diverse and is home to unique, threatened and potential bird species. Unfortunately, the area also faces threats that endanger avian survival through the loss of habitat due to deforestation, migratory agriculture, the cultivation of Erythroxylum coca and/or the timber industry, species trafficking and overhunting. As such, it is important to implement management plans for avian species, focusing on priority habitats or tourism schemes that guarantee avian conservation.
\end{abstract}

KEYWORDS: avifauna, conservation, habitats, interfluvium, threats. 


\section{INTRODUCCIÓN}

El interfluvio Napo-Putumayo-Amazonas, es una vasta selva que alberga una megabiodiversidad de flora y fauna silvestre y una particular riqueza étnica y cultural (Gilmore et al., 2010). Se han realizado diversos estudios sobre la avifauna que habita en esta zona, siendo ésta considerada como indicadora de la salud de los ecosistemas, además, los estudios de la ornitofauna por hábitats nos ayudan a comprender los patrones de distribución de las aves. Las aves son fáciles de detectar, su muestreo es relativamente fácil y económico de realizar y poseen una taxonomía bien conocida y relativamente estable (Stotz et al., 1996; Zaccagnini et al., 2011; Birdlife International, 2018).

Los estudios avifaunísticos en el interfluvio se orientaron a generar información de localidades específicas, careciéndose de una fuente informativa a escala regional que permita conocer la diversidad de aves que alberga este importante sector de la Amazonía peruana, diversidad que generalmente está condicionada por múltiples factores como su ubicación geográfica o las características propias del ambiente.

Los estudios realizados en este sector permitieron conocer especies que están restringidas a esta zona de la Amazonía peruana, especies que han ampliado su distribución hacia este sector y registros notables (Stotz \& Pequeño, 2004; Stotz \& Mena, 2008; Stotz \& Díaz, 2010, 2011; Stotz \& Ruelas, 2013; Stotz et al., 2016; Vásquez-Arévalo \& Díaz, 2019), evidenciando que existe una riqueza diferenciada de aves en el bosque inundable y bosque de tierra firme en la cuenca del Putumayo (Stotz \& Díaz, 2010; Vásquez-Arévalo \& Díaz, 2019).

El estudio se realizó con la finalidad de dar a conocer la riqueza ornitológica asentada a una escala regional en el interfluvio Napo-PutumayoAmazonas, la composición ornitológica según los tipos de hábitats, las especies amenazadas que alberga y las principales amenazas a las que están expuestas las aves en este sector, de esta manera se comprenderán mejor los patrones de diversidad y distribución de las aves, especies claves o especies indicadoras de hábitats con características particulares en nuestra Amazonía.

Está información será de gran utilidad para la toma de decisiones tanto a nivel local, regional y nacional, dirigidas al establecimiento de estrategias de conservación de hábitats prioritarios, zonificación ecológica y económica y programas de manejo, que permitan aprovechar los recursos de manera sostenible en beneficio de la vida silvestre y las comunidades locales. Además, gran parte de la Amazonía peruana es un universo desconocido por descubrir, por lo que este estudio motivará el desarrollo de futuras investigaciones sobre la riqueza ornitológica de la Amazonía peruana.

\section{COLECTA DE DATOS}

\section{ÁREA DE ESTUDIO}

La zona del interfluvio Napo-PutumayoAmazonas se encuentra ubicada al noreste del departamento de Loreto, es de clima tropical, con una temperatura ambiental promedio de $26,3^{\circ} \mathrm{C}$, que oscila de $20,9^{\circ} \mathrm{C}$ a $31,7^{\circ} \mathrm{C}$, la humedad relativa mensual es del 88 \% y la precipitación promedio mensual fluctúa de los 208 a los 309 mm (ClimateData, 2019). La temporada de vaciante comienza en agosto y culmina en febrero, su nivel más bajo se registra durante el mes de noviembre y la creciente se inicia en el mes de marzo, con su pico máximo entre mayo y julio (Servicio de Hidrografía y Navegación de la Amazonía, 2019).

Se analizaron los datos de los inventarios realizados en los ríos Ampiyacu, Apayacu, Yaguas, Cotuhé, Ere, Campuya y Algodón (Stotz \& Pequeño, 2004; Stotz \& Díaz, 2011; Stotz \& Ruelas, 2013; 


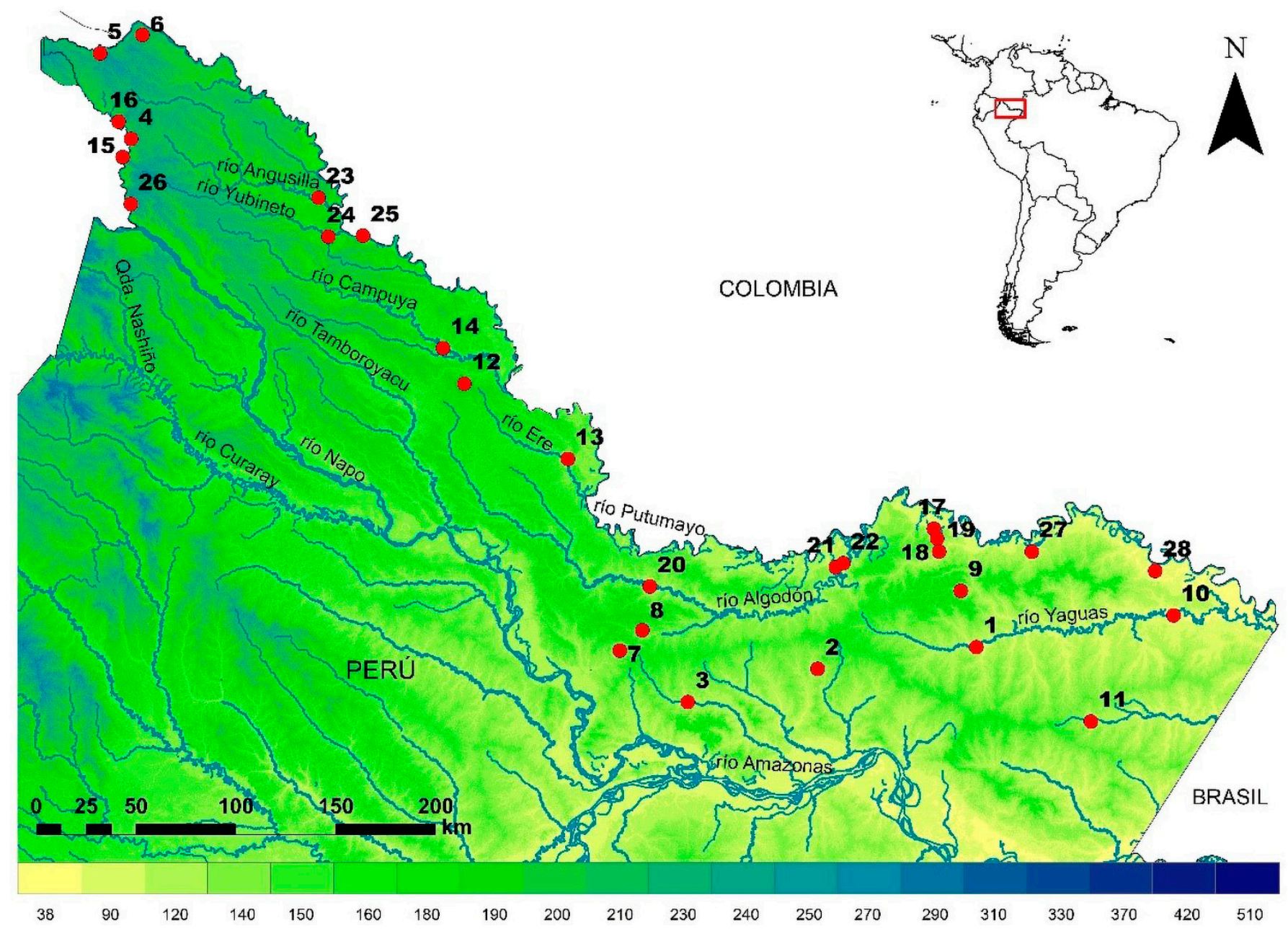

Figura 1. Mapa de ubicación de los estudios compilados de la avifauna en el interfluvio Napo-Putumayo-Amazonas. La leyenda de los números se puede ver en la tabla 1.

Stotz et al., 2016), en el interfluvio de los ríos Napo y Putumayo de la frontera de Perú, Ecuador y Colombia (Stotz \& Mena, 2008), en la propuesta del Área de Conservación Regional Maijuna (Stotz \& Díaz, 2010) y en el Complejo Lagunar Lagartococha (Naranjo et al., 2016). Se analizaron también los datos de los estudios de evaluación poblacional de las aves en tres comunidades de la cuenca alta del Putumayo (Vásquez-Arévalo \& Díaz, 2019), en el río Aguarico (Angulo-Perez et al., 2019) y en dos comunidades de la cuenca baja del Putumayo (Angulo-Perez et al., en prensa) (Figura 1) (Tabla 1).

\section{MÉTODOS}

Se realizó una exhaustiva revisión bibliográfica de los estudios ornitológicos de la zona, además se usaron bases de datos de los estudios realizados mediante transectos y redes de neblina. Estas informaciones permitieron conocer la composición ornitológica por tipo de hábitat en la zona de estudio.

\section{REVISIÓN BIBLIOGRÁFICA}

La revisión bibliográfica permitió conocer la riqueza de aves en el interfluvio Napo- 
Tabla 1: Lista de fuentes de información, lugares de muestreo, coordenadas de ubicación y hábitat dominante en los estudios en aves realizados en el interfluvio Napo-Putumayo-Amazonas.

\begin{tabular}{|c|c|c|c|c|c|}
\hline Autor & Código & Localidad & Latitud & Longitud & $\begin{array}{l}\text { Hábitat } \\
\text { dominante }\end{array}$ \\
\hline \multirow{3}{*}{$\begin{array}{l}\text { Stotz \& Pequeño, } \\
2004\end{array}$} & 1 & Yaguas & $-2,8648612$ & $-77,4150277$ & Inundable \\
\hline & 2 & Maronal & $-2,9656389$ & $-72,1278611$ & Tierra firme \\
\hline & 3 & Apayacu & $-3,1166667$ & $-72,7125$ & Tierra firme \\
\hline \multirow{3}{*}{ Stotz \& Mena, 2008} & 4 & Redondococha & $-0,5713056$ & $-75,2192222$ & Tierra firme \\
\hline & 5 & Güeppí & $-0,1846944$ & $-75,3589722$ & Tierra firme \\
\hline & 6 & Aguas Negras & $-0,1004445$ & $-75,1679722$ & Tierra firme \\
\hline \multirow{2}{*}{ Stotz \& Díaz, 2010} & 7 & Curupa & $-2,8850278$ & $-73,0186667$ & Tierra firme \\
\hline & 8 & Piedras & $-2,7927501$ & $-72,9174723$ & Tierra firme \\
\hline \multirow{3}{*}{ Stotz \& Diaz, 2011} & 9 & Choro & $-2,6106111$ & $-71,48575$ & Tierra firme \\
\hline & 10 & Cachimbo & $-2,7183055$ & $-70,5291944$ & Inundable \\
\hline & 11 & Alto Cotuhé & $-3,1987778$ & $-70,8990278$ & Inundable \\
\hline \multirow{3}{*}{ Stotz \& Ruelas, 2013} & 12 & Ere- Algodón & $-1,6790278$ & $-73,7196944$ & Tierra firme \\
\hline & 13 & Bajo Ere & $-2,0187223$ & $-73,2537222$ & Inundable \\
\hline & 14 & Medio Campuya & $-1,5176112$ & $-73,8161667$ & Tierra firme \\
\hline \multirow{2}{*}{ Naranjo et al., 2016} & 15 & Lagartococha & $-0,6531368$ & $-75,2570726$ & Inundable \\
\hline & 16 & Laguna Tambo II & $-0,493525$ & $-75,275363$ & Inundable \\
\hline \multirow{6}{*}{ Stotz et al., 2016} & 17 & Quebrada Bufeo & $-2,3306111$ & $-71,6075278$ & Tierra firme \\
\hline & 18 & Quebrada Agua Blanca & $-2,3750556$ & $-71,5928611$ & Tierra firme \\
\hline & 19 & Quebrada Mutún & $-2,4335$ & $-71,5838056$ & Tierra firme \\
\hline & 20 & Medio Algodón & $-2,5950556$ & $-72,8840555$ & Inundable \\
\hline & 21 & Bajo Algodón & $-1,1145362$ & $-11,5940454$ & Inundable \\
\hline & 22 & Chave Cocha & $-2,4877778$ & $-72,0141944$ & Inundable \\
\hline \multirow{3}{*}{$\begin{array}{l}\text { Vásquez-Arévalo \& } \\
\text { Díaz, } 2019\end{array}$} & 23 & Mashunta & $-0,8375074$ & $-74,3765237$ & Inundable \\
\hline & 24 & Santa Rita & $-1,0134187$ & $-74,3326192$ & Inundable \\
\hline & 25 & Nuevo Jerusalén & $-1,0090508$ & $-74,1756306$ & Tierra firme \\
\hline $\begin{array}{l}\text { Angulo-Perez et al., } \\
2019\end{array}$ & 26 & Río Aguarico & $-0,8661334$ & $-75,220675$ & Tierra firme \\
\hline \multirow{2}{*}{$\begin{array}{l}\text { Angulo-Perez et al., } \\
\text { (en prensa) }\end{array}$} & 27 & Remanso & $-2,4329639$ & $-71,1675$ & Tierra firme \\
\hline & 28 & Tres Esquinas & $-2,5180705$ & $-70,6127032$ & Inundable \\
\hline
\end{tabular}


Putumayo-Amazonas, pues al ser un territorio amplio, se compilaron numerosos datos de los estudios realizados en esta zona. Se compilaron diez estudios sobre la avifauna en la zona del interfluvio Napo-Putumayo-Amazonas, entre inventarios rápidos (Stotz \& Pequeño, 2004; Stotz \& Mena, 2008; Stotz \& Díaz, 2010, 2011; Stotz \& Ruelas, 2013; Naranjo et al., 2016; Stotz et al., 2016) y estudios poblacionales de aves (AnguloPerez et al., 2019; Angulo-Perez et al., en prensa; Vásquez-Arévalo \& Díaz, 2019).

\section{TRANSECTOS}

El método de transectos fue de gran utilidad para evaluar la composición de la avifauna según los tipos de hábitats principales presentes en la zona de estudio (colina baja, terraza alta, terraza media, varillal pantanoso y aguajal mixto) (AlvaVela et al., en prensa; Zárate et al., 2019a, 2019b).

Se utilizó la base de datos de los estudios poblacionales de aves en la cuenca alta (VásquezArévalo \& Díaz, 2019) y cuenca baja del Putumayo (Angulo-Perez et al., en prensa). El método de evaluación por transectos consistió en la búsqueda de aves a lo largo de transectos entre 3 a $5 \mathrm{~km}$ de distancia, entre las 06:00 y las 13:00 horas, registrándose todas las especies y número de individuos por observación directa y/o audición, teniendo en cuenta el tipo de hábitat evaluado, contando con una grabadora para el registro de las vocalizaciones de especies que no pudieron ser identificadas in situ.

\section{REDES DE NEBLINA}

La captura de aves del sotobosque mediante redes de neblina es un método complementario usado en la evaluación poblacional de aves en el río Aguarico (Angulo-Perez et al., 2019), que permitió registrar aquellas especies que no pudieron ser registradas por el método de transectos. Este método consistió en la instalación de 15 redes de neblina de 12 × 2,5 m, dispuestas en tres series de
5 redes, con una distancia de 500 m entre cada serie. Estas redes fueron abiertas desde las 06:00 a las 13:00 horas, con revisiones periódicas cada media hora o una hora.

\section{IDENTIFICACIÓN DE ESPECIES}

Las especies fueron identificadas in situ, utilizando el libro de Aves del Perú (Schulenberg et al., 2010) y las vocalizaciones grabadas que no pudieron ser reconocidas en campo, comparándolas con el banco de vocalizaciones de aves en línea xeno-canto (www.xeno-canto.org). La nomenclatura científica sigue al South American Classification Committee-SACC (Remsen et al., 2020) y a la Lista de las Aves del Perú, actualizada al 04 de agosto de 2020 (Plenge, 2020).

\section{ANÁLISIS ESTADÍSTICO}

Se generó una base de datos de las especies, teniendo en cuenta su orden, familia, género, zona de estudio, coordenadas geográficas y autores del estudio donde se registraron. De igual manera, se generó otra base de datos de la evaluación de aves a través de transectos por tipos de hábitats, considerando principalmente el número de individuos por especie en cada tipo de hábitat evaluado. Los registros de un género, que no lograron ser identificados, o aquellas especies cuya presencia no presentaba evidencias en esta zona, fueron eliminados para evitar sobrestimar la riqueza de especies.

La riqueza específica observada se estimó a través del conteo de especies válidas y la riqueza específica esperada fue calculada con el estimador no paramétrico Chao-2, utilizando el software Species Diversity and Richness 4.1.2 (SDR4) (Seaby \& Henderson, 2006). Se aplicó el método de rarefacción para comparar la riqueza por tipo de hábitat (colina baja, terraza alta, terraza media, varillal pantanoso y aguajal mixto) 
y la riqueza esperada por hábitat se obtuvo con el índice no paramétrico de Chao-1 utilizando PAST 3.15 (Hammer et al., 2001) y graficada mediante SigmaPlot 11.0 (Systat Software Inc. 2008).

La similitud entre hábitats se midió mediante el análisis de agrupamiento con el índice de Bray Curtis, utilizando PAST 3.15 (Hammer et al., 2001).

Las especies únicas se identificaron en base a su distribución geográfica restringida, dotando de gran importancia a la zona del interfluvio NapoPutumayo-Amazonas.

Adicionalmente, se identificaron las especies de aves que se encuentran bajo alguna categoría de amenaza en el Perú, según el Decreto Supremo $\mathrm{N}^{\circ}$ 004-2014-MINAGRI (MINAGRI, 2014) y la Lista Roja de la Unión Internacional para la Conservación de la Naturaleza-IUCN (IUCN, 2020) y aquellas especies que están incluidas en los apéndices I y II de la Convención sobre el Comercio Internacional de Especies Amenazadas de Fauna y Flora Silvestre-CITES (CITES, 2017).

Las amenazas y recomendaciones para la conservación de las aves en la zona del interfluvio Napo-Putumayo-Amazonas se identificaron por puntos de evaluación y a través de la información proporcionada por cada uno de los estudios realizados.

\section{RESULTADOS}

RIQUEZA EN EL INTERFLUVIO NAPOPUTUMAYO-AMAZONAS

Los estudios realizados desde el año 2004 al 2019 registraron una riqueza de 558 especies distribuida en 24 órdenes, 63 familias y 359 géneros (Apéndice 2). Las familias con mayor riqueza de especies fueron Tyrannidae, Thamnophilidae y Furnariidae, con 59, 57 y 43 especies, respectivamente. Según el estimador no para- métrico de Chao-2, en los sectores evaluados del interfluvio Napo-Putumayo-Amazonas se registró el $93 \%$ de las especies esperadas (602 especies) (Figura 2).

\section{RIQUEZA EN LOS HÁBITATS}

Los cinco hábitats evaluados (bosque de aguajal mixto, colina baja, terraza alta, terraza media y varillal pantanoso o hidromórfico) presentaron diferentes valores de riqueza de aves. El bosque de colina baja alberga la mayor riqueza de especies, mientras que el bosque de terraza media alberga la menor riqueza. En el bosque de colina baja se registraron 183 especies, representando el $91 \%$ de las especies esperadas (201 especies), mientras que en el bosque de aguajal mixto, varillal pantanoso y terraza alta se registraron 161,135 y 126 especies, respectivamente, representando el 91, 93 y $90 \%$ de las especies esperadas $(177,145$ y 140 especies, respectivamente). En el bosque de terraza media se registraron 76 especies, que conforman el $87 \%$ de las especies esperadas (87 especies) (Figura 3).

\section{FAMILIAS EN LOS HÁBITATS}

Las familias Thamnophilidae, Tyrannidae y Furnariidae estuvieron mejor representadas en casi todos los tipos de hábitats evaluados.

La familia Thamnophilidae posee mayor riqueza de especies en todos los hábitats evaluados. En el bosque de aguajal mixto, varillal pantanoso y terraza alta, las familias más representativas fueron Thamnophilidae $(27,22$ y 24 especies), Tyrannidae (19, 15 y 17 especies) y Furnariidae (17, 14 y 9). En el bosque de terraza media, las familias más representativas fueron Thamnophilidae con 17 especies, Tyrannidae con 10 especies y Psittacidae con 7 especies; mientras que, en colina baja, las más representativas fueron Thamnophilidae con 30 especies, Furnariidae con 21 especies y Tyrannidae con 19 especies (Figura 4). 

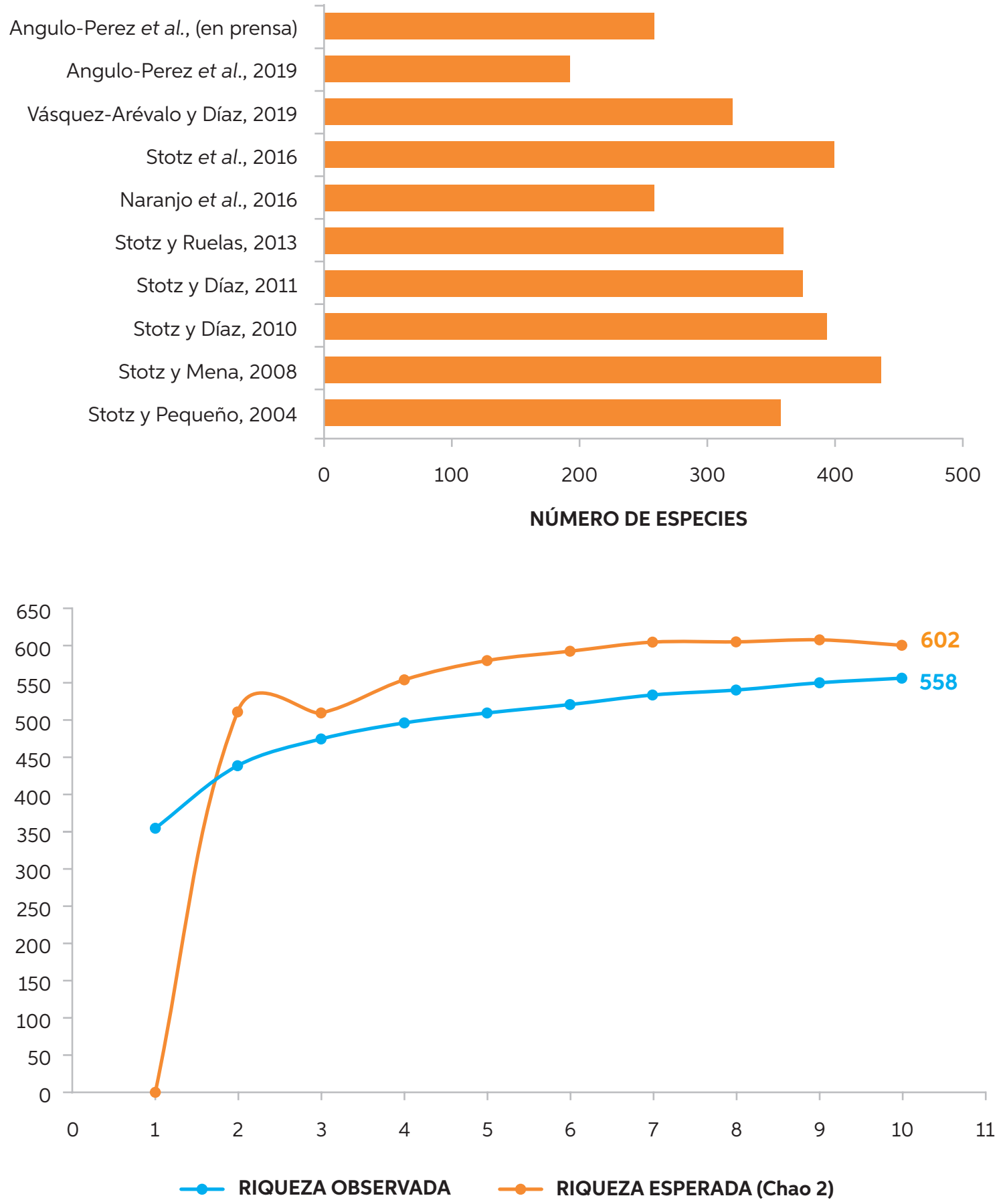

Figura 2. A. Número de especies de aves registradas por estudio del 2004 al 2019.(superior). Riqueza observada y esperada en el interfluvio Napo-Putumayo-Amazonas (inferior). 


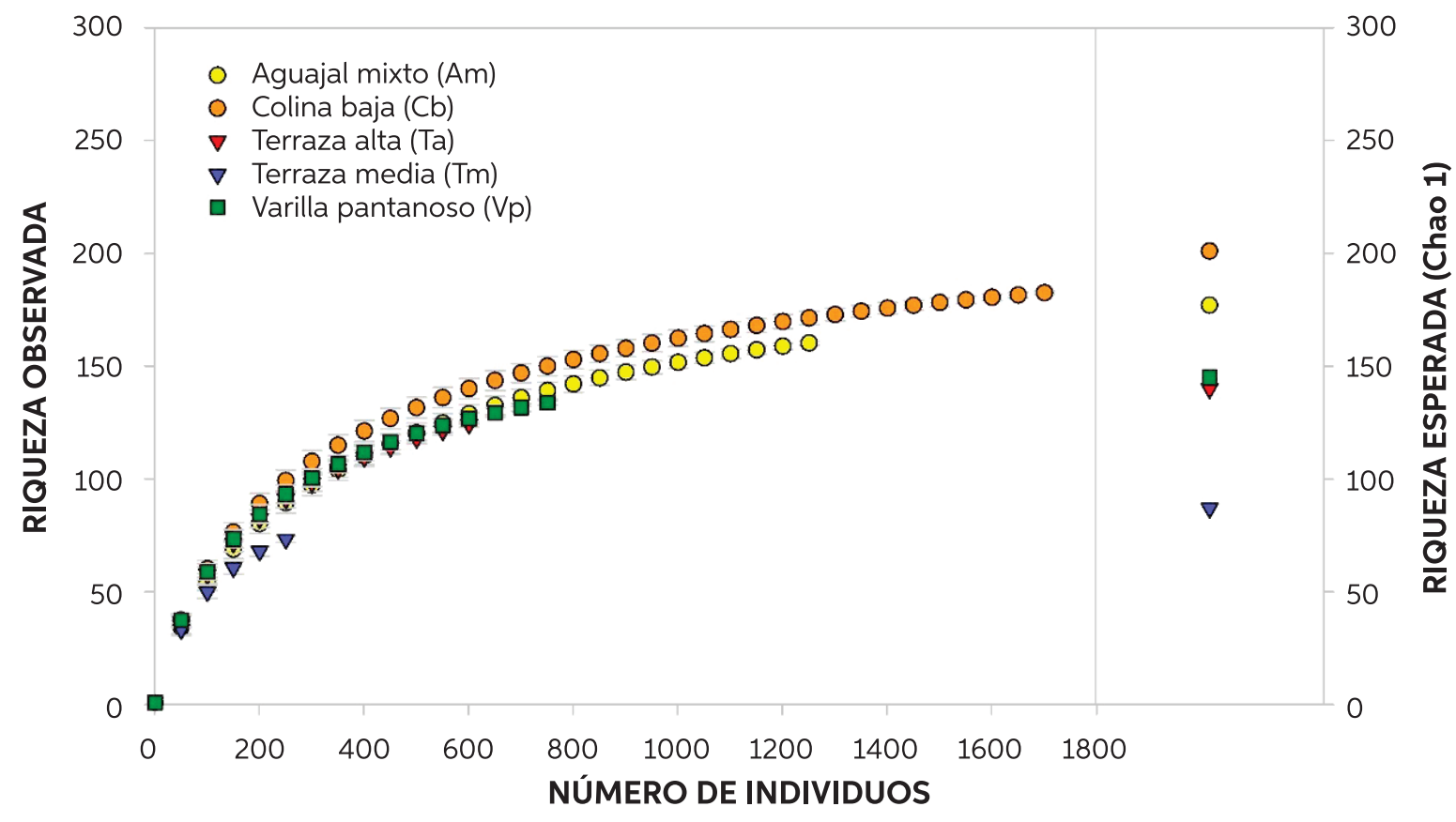

Figura 3. Riqueza específica observada (izquierda) y esperada de aves usando el estimador no paramétrico de Chao-1 (derecha) en los bosques de aguajal mixto, colina baja, terraza alta, terraza media y varillal pantanoso.

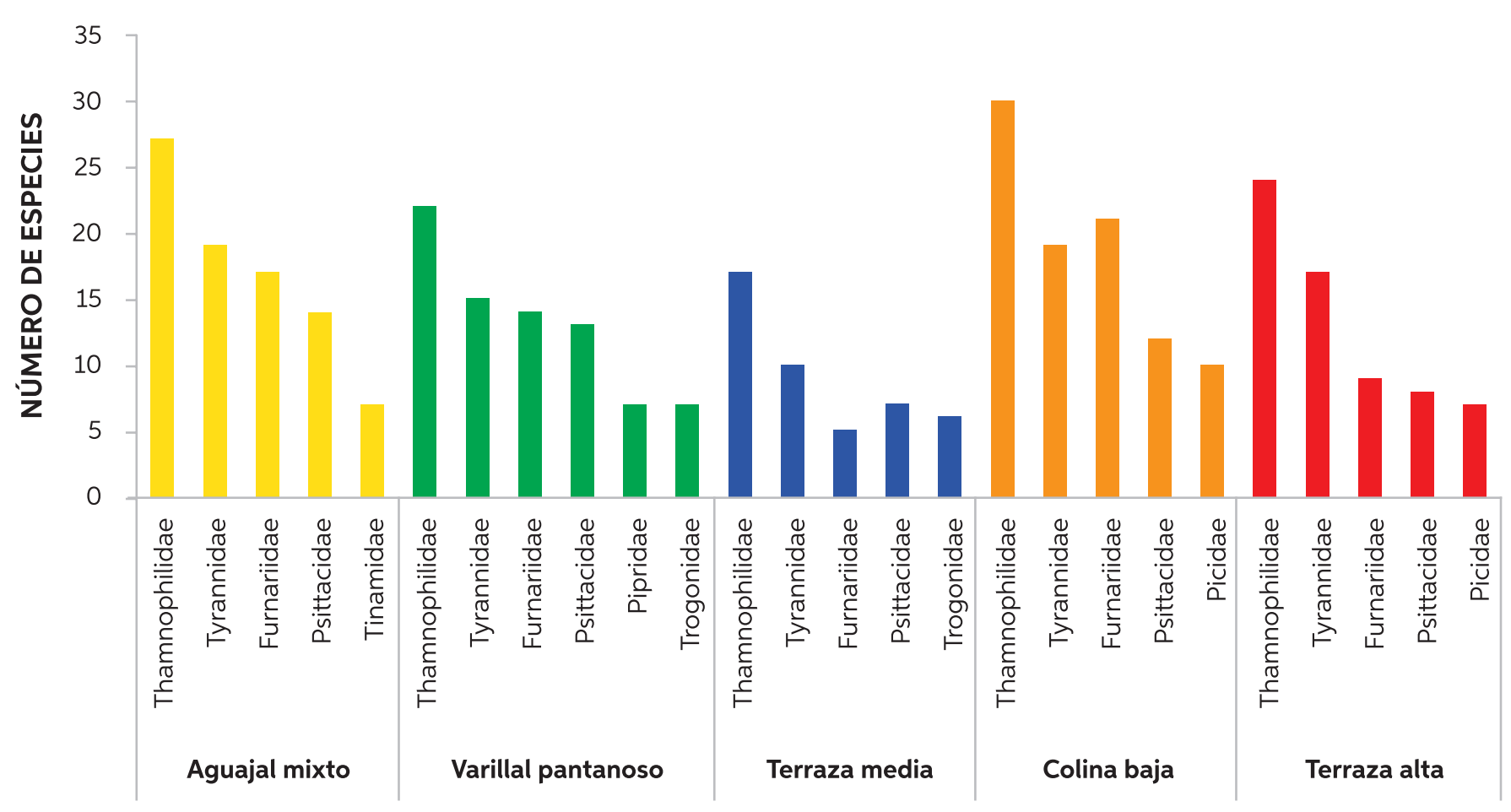

Figura 4. Riqueza de especies de aves por familia, según los tipos de hábitats en el interfluvio Napo-Putumayo-Amazonas. 


\section{SIMILITUD ENTRE HÁBITATS}

El análisis de agrupamiento de los transectos de muestreo por tipo de hábitat formó dos grandes grupos compuestos por hábitats de tierra firme (principalmente colina baja, terraza alta y terraza media) y hábitats inundables (varillal pantanoso y aguajal mixto). El primer grupo se dividió en dos subgrupos; en el primer subgrupo se encuentran los transectos que recorrieron colina baja y terraza alta; y en el segundo subgrupo los transectos que recorrieron terraza alta, colina baja, terraza media y también algunos transectos de varillal pantanoso. Esto demuestra que hay una diferenciación de especies por hábitat inundable y de tierra firme y que las terrazas medias son hábitats transicionales muy importantes que albergan especies de aves de tierra firme e inundable (Figura 5).

\section{ESPECIES ÚNICAS DEL INTERFLUVIO NAPO- PUTUMAYO-AMAZONAS}

Las especies únicas que poseen una distribución restringida y que dotan de importancia a la zona de estudio son Herpsilomus sp., un hormiguerito que actualmente se encuentra en proceso de estudio, registrado en el río Ampiyacu y Apayacu en hábitats de suelos pobres, con una distribución restringida al interfluvio de los ríos Napo y Putumayo; Percnostola rufifrons jensoni, especialista de suelos pobres, que se encuentra restringida a la zona del interfluvio NapoPutumayo, al igual que Euchrepomis spodioptila,

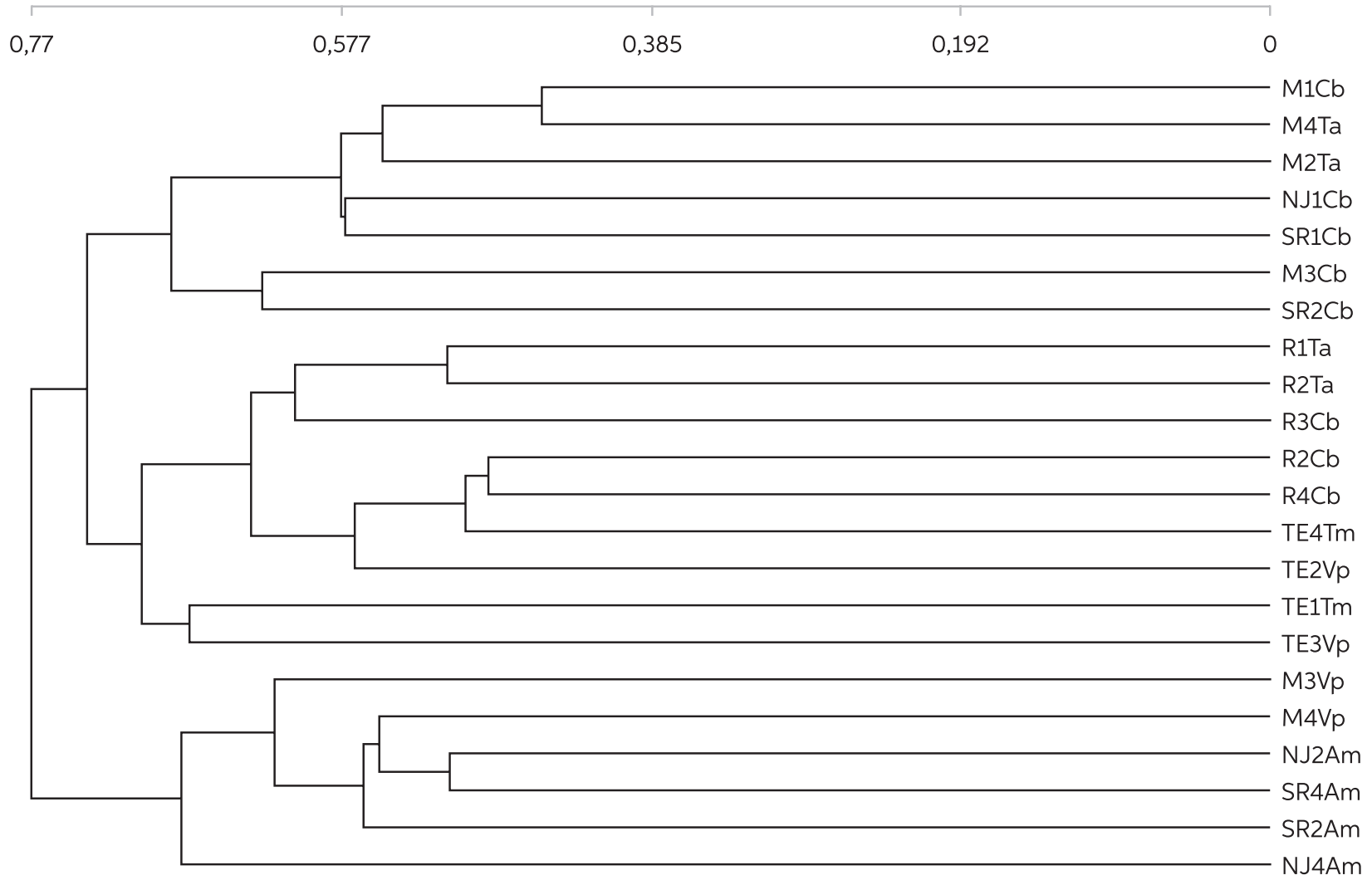

Figura 5. Similitud de especies entre hábitats del interfluvio Napo-Putumayo-Amazonas usando el índice de BrayCurtis. Donde: Comunidades de estudio: M: Mushunta, NJ: Nueva Jerusalén, SR: Santa Rita, R: Remanso, TE: Tres Esquinas. Transectos estudiados: 1: Transecto 1, 2: Transecto 2, 3: Transecto 3, 4: Transecto 4. Tipos de hábitats: Cb: Colina baja, Ta: Terraza alta, Tm: Terraza media, Vp: Varillal pantanoso, Am: Aguajal mixto. 
con rango limitado de distribución al norte de la Amazonía y otras especies como Microbates collaris, Campylorhamphus procurvoides y Grallaria varia.

\section{ESPECIES AMENAZADAS A NIVEL NACIONAL Y} MUNDIAL

Según la legislación peruana, cinco especies de aves del interfluvio Napo-Putumayo-Amazonas se encuentran dentro de alguna categoría de amenaza, una en Peligro Crítico (Crax globulosa) y cuatro en la categoría Vulnerable (Mitu salvini, Crypturellus duidae, Harpia harpyja y Nyctibius leucopterus) (MINAGRI, 2014).

En la lista roja de la Unión Internacional para la Conservación de la Naturaleza y los Recursos Naturales (IUCN), se incluyen siete especies en categoría de amenaza, Crax globulosa en la categoría En Peligro (EN) y Patagioenas subvinacea, Agamia agami, Ramphastos tucanus, Ramphastos vitellinus, Touit huetii y Myrmoborus lugubris en la categoría Vulnerable (VU).

Por otro lado, 89 especies de aves se ubican dentro de la lista de especies que pueden ser afectadas por el comercio, según la Convención sobre el Comercio Internacional de Especies Amenazadas de Fauna y Flora Silvestre (CITES). De estas especies, 87 se encuentran en el apéndice II y dos especies en el apéndice I (CITES, 2017) (Apéndice 1).

\section{AMENAZAS Y RECOMENDACIONES PARA LA} CONSERVACIÓN

La pérdida de hábitats por agricultura migratoria, por siembra de Erythroxylum coca o tala de árboles por la industria maderera, el tráfico de especies y la sobrecaza de algunas especies de aves, son las principales causas que ponen en peligro a la avifauna del interfluvio NapoPutumayo-Amazonas. En el sector de los ríos Ere, Campuya y Algodón, en la propuesta ACR Maijuna y el sector de Yaguas, la avifauna se ve afectada principalmente por la pérdida de la cobertura arbórea y en menor grado por la sobrecaza. En la zona del río Ampiyacu, Apayacu, Yaguas y Medio Putumayo, la principal amenaza para la avifauna es la pérdida de hábitats por la deforestación; y en el río Algodón son la deforestación, la sobrecaza y el cambio en el uso de los suelos, en especial de las islas y bosques inundados circundantes al río Putumayo. En el sector de Cuyabeno-Güeppi, la deforestación, la sobrecaza y la perturbación de hábitats son las principales causas que amenazan a la avifauna; y en el alto Putumayo se destaca como mayor amenaza el sembrío de Erythroxylum coca, que puede afectar a algunas especies de aves, por la deforestación provocada por esta actividad.

La implementación de planes de manejo y conservación en este sector de la Amazonía, en colaboración con las comunidades locales, debe ser prioritaria para asegurar la caza sostenible y mantener saludables a las poblaciones de aves, sin que estas se vean afectadas en un futuro.

De igual manera, es necesario establecer planes de conservación para la protección de determinados tipos de bosques que albergan especies especialistas o implementar planes de manejo específicos para la protección estricta de especies amenazadas, pues la alteración de sus hábitats puede repercutir negativamente en su supervivencia. La zona del interfluvio alberga muchas especies de aves que son de interés para el aviturismo mundial, por lo que la implementación de planes turísticos con la participación de las comunidades locales sería de gran apoyo para la economía familiar de esta zona.

\section{DISCUSIÓN}

La riqueza ornitológica en el interfluvio es alta, con 558 especies, representando más del 50\% de total de especies que se estiman para Loreto (Pitman et al., 2013), mostrando a esta zona 
como clave para las aves amazónicas. A pesar de haber sido explorada en las últimas décadas, todavía existen zonas sin explorar, como a lo largo de los grandes ríos Amazonas y Putumayo e islas fluviales, que podrían albergar especies por descubrir e incrementar la riqueza en este sector de la Amazonía. El mosaico de diferentes tipos de suelo y vegetación son bien definidos en esta zona norte de Loreto (Gentry \& Ortiz, 1993), cuyo origen está vinculado con la dinámica del levantamiento de la cordillera de los Andes, lo que puede explicar la gran diversidad de aves que alberga el interfluvio, existiendo especies de aves asociadas a un tipo de hábitat determinado.

Las características diferenciadas de los hábitats influyen en las aves. Numerosos estudios han evidenciado que la estructura de la vegetación y la composición florística son dos componentes del hábitat que influyen marcadamente en la composición y la abundancia de las aves, tanto en el ambiente natural como en el urbano, cuyas características pueden condicionar un ambiente propicio donde encuentren las condiciones básicas para su alimentación, descanso y reproducción (Cody, 1981; Cueto, 2006; AnguloPerez et al., 2017; Pollack et al., 2018; SalasCorrea \& Mancera-Rodríguez, 2018). De los hábitats evaluados en el interfluvio, el bosque de colina baja albergó mayor riqueza de aves en comparación a otros tipos de hábitats, debido posiblemente a que este tipo de bosque alberga una mayor diversidad de especies de plantas (Zárate et al., 2019a, 2019b), lo que propiciaría el establecimiento de una mayor diversidad de especies de aves.

La composición de aves difiere entre el hábitat inundable y de tierra firme (Stotz \& Díaz, 2011; Vásquez-Arévalo \& Díaz, 2019), pero los distintos tipos de hábitats inundables (varillal pantanoso y aguajal mixto) y de tierra firme (colina baja, terraza alta y terraza media) pueden compartir especies entre sí. El bosque de terraza media es un tipo de bosque transicional que generalmente representa las partes altas de las áreas inundables y alberga una vegetación compartida propia del bosque inundable y de tierra firme, por lo que juega un papel importante al albergar posiblemente especies de aves más especializadas que todavía han sido poco investigadasy que puedan adaptarse a los cambios en el hábitat según la estacionalidad hidrológica, lo que explicaría la poca riqueza de aves registradas en este tipo de hábitat.

En los cinco tipos de hábitats estudiados en el interfluvio Napo-Putumayo-Amazonas, las familias mejor representadas en cuanto a riqueza de especies fueron Thamnophilidae, Tyrannidae y Furnariidae, debido a que estas familias son las más diversificadas, cuyas especies están adaptadas a diversos tipos de hábitats existentes; además, las especies de estas familias forman bandadas mixtas y se desplazan por el sotobosque en busca de pequeños insectos, artrópodos o frutos que les puede proveer el bosque.

El interfluvio Napo-Putumayo-Amazonas alberga un conjunto de especies de aves únicas. Por ejemplo, el Hormiguero de Cabeza Negra (Percnostola rufifrons jensoni) y la posible nueva especie de Hormiguerito (Herpsilochmus sp.) son aves especialistas de bosques de suelos pobres, consideradas únicas del interfluvio NapoPutumayo-Amazonas en Perú, de manera conjunta con otras especies que presentan una distribución restringida a esta zona, como Euchrepomis spodioptila, Microbates collaris, Campylorhamphus procurvoides y Grallaria varia, conformando un grupo de aves con gran potencial en el aviturismo nacional e internacional, actividad que se desarrolla en otras zonas de la región Loreto, como en la Reserva Nacional Allpahuayo Mishana, muy conocida por la riqueza ornitológica que posee (Álvarez, 2007); además dicha actividad apoyaría a la conservación y revaloración de la avifauna 
de esta zona, generando puestos de trabajo a los pobladores locales, mejorando así su economía familiar (Stotz et al., 2016).

Los crácidos son un grupo de aves importantes dentro de los ecosistemas amazónicos y son fuente de alimentos en muchas comunidades en el interfluvio Napo-Putumayo-Amazonas (Stotz et al., 2016; Brooks \& Strahl, 2000). Actualmente, especies como Mitu salvini y Crax globulosa se encuentran dentro de algún grado de amenaza a nivel nacional e internacional. La situación actual de C. globulosa es crítica, debido a que se encuentra amenazada por la sobrecaza y destrucción de su hábitat (Alarcón-Nieto \& Palacios, 2008; Yahuarcani et al., 2009, Brooks \& Strahl, 2000). Los registros existentes de esta especie en el interfluvio Napo-PutumayoAmazonas provienen de los comentarios de los pobladores locales, que mencionan haberla visto o escuchado en algunas islas grandes, siendo estas islas uno de los últimos refugios del Perú en los cuales habitaría esta especie (Stotz et al., 2016). Llevar a cabo estudios específicos sobre el estado poblacional, ecología y otros aspectos biológicos de Crax globulosa sería de gran importancia para generar información útil que servirá en un futuro para la implementación de planes de conservación del hábitat y poblaciones de C. globulosa en el interfluvio (Yahuarcani et al., 2009; Alarcón-Nieto \& Palacios, 2008; Aranibar-Rojas \& Hennessey, 2006; Brooks \& Strahl, 2000). De igual manera, desarrollar un programa de educación ambiental orientado a trabajar con las comunidades locales puede ser una estrategia clave para contribuir con la conservación de la biodiversidad de este sector (González, 2002)

Las especies Notarchus ordii y Crypturellus duidae, consideradas como especialistas estrictas de los bosques sobre arena blanca (Álvarez et al., 2013), fueron registradas en el interfluvio Napo-
Putumayo-Amazonas, ampliando su rango de distribución a esta zona. $N$. ordii y $C$. duidae fueron registradas en un varillal pantanoso (Vásquez \& Díaz, 2019), lo cual indicaría que estas especies no solamente están presentes en bosques sobre arena blanca, sino en diferentes tipos de bosques de suelos pobres (Stotz et al., 2016).

Realizar estudios en los diversos hábitats que aún no han sido explorados en el interfluvio podría ser muy relevante, debido a que especies nuevas para la ciencia podrían ser descubiertas, pero también se podrían registrar otras especies para esta zona, con lo que se incrementaría la riqueza de aves a nivel nacional, como el caso del Corocoro Escarlata (Eudocimus ruber) que fue registrado por primera vez en el Perú en la cuenca alta del Putumayo, cuya distribución se ha ido ampliando hasta la periferia de la ciudad de Iquitos (Angulo et al. 2019; Ruiz-Ramos et al., 2020).

Lamentablemente, la avifauna que alberga el interfluvio se encuentra amenazada por la destrucción de hábitats por la deforestación, agricultura migratoria, la siembra de Erythroxylum coca, tala de árboles por la industria maderera, el tráfico de especies y la sobrecaza de algunas especies de aves, por ello debe ser una prioridad implementar planes de manejo o planes de conservación de especies o hábitats determinados que permitan un adecuado aprovechamiento de los recursos y brinden facilidades y apoyo a las comunidades locales para potenciar actividades económicas que no afecten la sostenibilidad de los recursos, iniciativa que debe desarrollarse articuladamente entre las instituciones competentes y las comunidades locales, estableciendo estrategias que permitan el mejoramiento de su calidad de vida y la conservación de los recursos naturales en este sector de la Amazonía peruana. 


\section{CONCLUSIONES}

El interfluvio Napo-Putumayo-Amazonas posee una gran riqueza ornitológica, albergando más del $50 \%$ de especies estimadas para Loreto, riqueza probablemente relacionada con el mosaico de tipos de suelos y tipos de hábitats presentes en esta zona. La riqueza de especies varía según los tipos de hábitats, siendo el bosque de colina baja el que alberga mayor riqueza de aves, debido posiblemente a la mayor riqueza de especies de plantas que posee este tipo de hábitat en relación a otros. La zona de estudio alberga hábitats claves para aves particulares, especies amenazadas o especies que dotan de importancia a la zona, por lo que es necesario establecer estrategias de conservación y aprovechamiento sostenible de los recursos que permitan mitigar las actividades que amenazan la supervivencia de estas especies, a través de un trabajo articulado entre las comunidades locales e instituciones competentes, con la finalidad de mejorar la calidad de vida de estos pobladores amazónicos.

\section{AGRADECIMIENTOS}

Este trabajo se realizó gracias al financiamiento del proyecto: Expedición Binacional PerúColombia: Inventarios de diversidad biológica en el Gran Putumayo, en el marco del programa presupuestal 144. Muchas gracias a todas las instituciones que realizaron los estudios en esta zona y a las comunidades nativas involucradas en la ejecución del estudio.

\section{REFERENCIAS BIBLIOGRÁFICAS}

Alarcón-Nieto, G.; Palacios, E. 2008. Estado de la población del pavón moquirrojo (Crax globulosa) en el bajo río Caquetá, Amazonía
Colombiana. Ornitología Neotropical, 19: 371376.

Alva-Vela, A.A.; Mozombite-Pinto, L.; PérezMacedo, C.P. (En prensa). Vegetación y flora. In: Aquino,R.; Díaz,J.;Zárate, R. (Eds). Biodiversidad de la cuenca baja del Putumayo, Perú. Instituto de Investigaciones de la Amazonía Peruana. Iquitos, Perú.

Álvarez, A. 2007. Reserva Nacional Allpahuayo Mishana: una joya natural al lado de Iquitos. Instituto de Investigaciones de la Amazonía Peruana. Proyecto BIODAMAZ Perú-Finlandia. Iquitos, Perú. 63pp.

Álvarez, A.; Metz, M.R; Fine, P.V.A. 2013. Habitat specialization by birds in western amazonian white-sand forest. Biotropical, 45(3): 365-372. DOI: https://doi.org/10.1111/btp.12020

Angulo, F.; Begazo, A.; Lane, D.F.; Plenge, M.A.; Stotz, D.F.; Ugarte, M.; Valqui, V.; Wallker, B. 2019. Reporte del Comité de Registros de Aves Peruanas (CRAP) del periodo 2017. Boletín de la Unión de Ornitólogos del Perú (UNOP).14(2): 51-62.

Angulo-Perez, N.C.; Armas-Silva, J.A.; ZárateGómez, R.; Pérez-Peña, P.E. 2017. Ecología urbana de aves: Relación de las plantas, clima y ruido con la biodiversidad de aves en la ciudad de Iquitos, Perú. Folia Amazónica, 26(2): 121-138. DOI: https://doi.org/10.24841/ fa.v26i2.427

Angulo-Perez, N.C.; Armas-Silva, J.A.; Gallardo, D.; Díaz, J. 2019. Aves. In: Pérez-Peña, P.E.; RamosRodríguez, M.C.; Díaz, J.; Zárate, R.; Mejía K. (Eds). Biodiversidad en las cuencas del Napo y Curaray, Perú. Instituto de Investigaciones de la Amazonía Peruana; Centro para el Desarrollo del Indígena Amazónico. Iquitos, Perú. p. 99126.

Angulo-Perez, N.C; Saboya Del Castillo, P.; ArmasSilva, J.A. (En prensa). Aves. In: Aquino, R.; Díaz, J.; Zárate, R. (Eds). Biodiversidad de la cuenca baja del Putumayo, Perú. Instituto 
de Investigaciones de la Amazonía Peruana. Iquitos, Perú. p. 76-108.

Aranibar-Rojas, H.; Hennerssey, A.B. 2006. Historia natural y estimación preliminar de la abundancia relativa mediante tazas de encuentros de Crax globulosa en un bosque de várzea en el departamento del Beni, Bolivia. Cotinga, 26(1): 32-25.

BirdLife International. 2018. Introducción: Importancia de las aves. In: Allinson, T.; Vovk, E.; Burfield, I.; Butchart, S.; Dale, A.; Heath, M. (Eds). El Estado de conservación de las aves del mundo: tomando el pulso de nuestro planeta. Bird Life International. Cambridge, Reino Unido. p. 6-16.

Brooks, D.M.; Strahl, S.D. 2000. Curassows, guans and chachalacas. Status survey and conservation acction plan for cracids 2000-2004. IUCN. Gland, Switzerland. 182pp.

CITES. 2017. Apéndices I, II y III. (https://cites. org/sites/default/files/notif/S-Notif-2016068-A.pdf). Acceso: 20/07/2020.

Climate-Data. 2019. Climate: El Estrecho. (https://es.climate-data.org/america-del-sur/ peru/loreto/el-estrecho-45827/). Acceso: 20/08/2020.

Cody, M.L. 1981. Habitat selection in birds: The roles of vegetation structure, competitors an productivity. BioScience, 31(2): 107-113. DOI: https://doi.org/10.2307/1308252

Cueto, V.R. 2006. Escalas en ecología: su importancia para el estudio de la selección de hábitat en aves. Hornero, 21(1): 1-13.

Gentry, A.H.; Ortiz, R. 1993. Patrones de composición florística en la Amazonía peruana. In: Kalliola, R.; Puhakka, M; Danjoy, W. (Eds). Amazonía peruana - vegetación húmeda tropical en el llano subandino. Universidad de Turku; Oficina Nacional de Evaluación de Recursos Naturales. Turku, Finlandia. p. 155-166.

Gilmore, M.P.; Vriesendorp, C.; Alverson, W.S.; del Campo, A.; von May, R.; López, C.; Ríos, S. 2010.
Perú: Maijuna. Rapid biological and social inventories, Report 22. The Field Museum. Chicago, Illinois. 328pp.

González, E. 2002. Educación Ambiental para la Biodiversidad: Reflexiones sobre conceptos y practices. Tópicos de Educación Ambiental, 4(11): 76-85.

Hammer, Ø.; Harper, D.A.T.; Ryan, P.D. 2001. Past: Paleontological statistics software package for Education and data analysis. Paleontología Electrónica, 4(1): 9.

IUCN. 2020. IUCN red list of threatened species. (http://www.iucnredlist.org). Acceso: 15/08/ 2020.

MINAGRI. 2014. Decreto Supremo Nº 004-2014MINAGRI: Decreto Supremo que aprueba la actualización de la lista de clasificación y categorización de las especies amenazadas de fauna silvestre legalmente protegidas. El Peruano, 520497-520504.

Naranjo, L.G.; Salinas, L.; Quinteros, K., Chiu, A.; Chota, S.; Talexio, G.; Tangoy, H.; Chimbo, D.; Macanilla, D.; Gómez, L. 2016. Caracterización ornitológica delcomplejolagunar Lagartococha. In: Usma, J.S.; Ortega, C.; Valenzuela, S.; Deza, J.; Rivas J. (Eds). Diversidad biológica y cultural del Corredor Trinacional de Áreas Protegidas La Paya-Cuyabeno-Güeppí Sekime. Colombia -Ecuador-Perú. WWF. Bogotá, Colombia. p. 290-308.

Pitman, N.; Gagliardi, G.; Jenkins, C. 2013. La biodiversidad de Loreto, Perú. El conocimiento actual de la diversidad de plantas $y$ vertebrados terrestres. Center for International Environmental Law (CIEL). Washington DC. 40pp.

Plenge, M.A. 2020. List of the birds of Peru / Lista de las aves del Perú. Última actualización: 04/08/2020. Unión de Ornitólogos del Perú. Boletín UNOP.(https://sites.google.com/site/ boletinunop/checklist). Acceso: 24/10/2020. 
Pollack, L.; Rodríguez, E.; Paredes, Y.; Gutiérrez, J.; Mora, M. 2018. Aves silvestres asociadas a la flora urbana del distrito de Trujillo, región La Libertad, Perú, 2016-2017. Arnaldoa, 25(1): 241-272. DOI: http://doi.org/10.22497/ arnaldoa.251.25114

Remsen, J.V.Jr.; Areta J.I.; Bonaccorso, E.; Claramunt, S.; Jaramillo, A.; Pacheco, J.F.; Robbins, M.B.; Stiles, F.G.; Stotz, D.F.; Zimmer, K.J. 2020. A classification of the bird species of South America. American Ornithological Society. Actualización: 13 agosto de 2020. (http://www.museum.lsu.edu/ Remsen/ SACCBaseline.htm). Acceso 15/08/2020.

Ruiz-Ramos, S.A.; Angulo-Perez, N.C.; Saboya del Castillo, P.; Gaviria Celis, J.L.; Díaz Alván, J. 2020. Registros documentados del Corocoro escarlata (Eudocimus ruber Linnaeus, 1758; Aves: Threskiornithidae) en Loreto, Perú. Ciencia Amazónica, 8 (1): 123-130. DOI: https://doi.org/10.22386/ca.v8i1.285

Salas-Correa, A.D.; Mancera-Rodríguez, N.J. 2018. Relaciones entre la diversidad de aves y la estructura de la vegetación en cuatro etapas sucesionales de bosque secundario, Antioquia, Colombia. Revista U.D.C.A Actualidad \& Divulgación Científica, 21(2): 519-529. DOI: https://doi.org/10.31910/rudca.v21. n2.2018.970

Seaby, R.M.H.; Henderson, P.A. 2007. Community analysis package 4.0. Pisces Conservation Ltd., Lymington, UK.

Schulenberg, T.S., Stotz, D.F.; Lane, D.F.; O'Neill, J.P.; Parker III, T.A. 2010. Aves de Perú, serie biodiversidad CORDIBI, 1. Lima, Perú. 660pp.

Servicio de Hidrografía y Navegación de la Amazonía. 2019. Avisos a los navegantes fluviales. Marina de Guerra del Perú. Iquitos, Perú. 41pp.

Stotz, D.F.; Fitzpatrick, J.W.; Parker III, T.A; Moskovits, D.K. 1996. Neotropical birds: Ecology and conservation. University of Chicago Press, Chicago, Illinois. 483pp.

Stotz, D.F; Pequeño, T. 2004. Aves. In: Pitman, N.; Smith, R.C.; Vriesendorp, C.; Moskovits, D.; Piana, R.; Knell, G; Wachter, T. (Eds). Perú: Ampiyacu, Apayacu, Yaguas, Medio Putumayo. Rapid biological and social inventories, Report 12. The Field Museum, Chicago Illinois. p. 7080; 242-253.

Stotz, D.F.; Mena, P. 2008. Aves. In: Alverson, W.S.; Vriesendorp, C.; del Campo, A.; Moskovits, D.K.; Stotz, D.F.; García, M.; Borbor, L.A. (Eds). Ecuador, Perú: Cuyabeno-Güeppí. Rapid biological and social inventories, Report 20. The Field Museum, Chicago, Illinois. p. 96-105; 324-351.

Stotz, D.F.; Díaz, J. 2010. Aves. In: Gilmore, M. P.; Vriesendorp, C.; Alverson, W. S.; del Campo, Á.; von May, R.; López Wong, C.; Ríos Ochoa, S. (Eds). Perú: Maijuna. Rapid Biological and Social Inventories, Report 22. The Field Museum, Chicago, Illinois. p. 81-90.

Stotz, D.F.; Díaz, J. 2011. Aves. In: Pitman, N.; Vriesendorp, C.; Moskovits, D.K.; von May, R.; Alvira, D.; Wachter, T.; Stotz, D.F.; del Campo, A. (Eds). Perú: Yaguas - Cotuhé. Rapid biological and social inventories, Report 23. The Field Museum, Chicago, Illinois. p. 116-125; 336355.

Stotz, D.F; Ruelas, E. 2013. Aves. In: Pitman, N.; Ruelas, E.; Vriesendorp, C.; Stotz, D.F.; Watchter, T.; del Campo, A.; Alvira, D.; Rodríguez, B.; Smith, R.C.; Sáenz, A.R; Soria, P. (Eds). Perú: Ere Campuya - Algodón. Rapid biological and social inventories, Report 25. The Field Museum, Chicago, Illinois. p. 114-120; 362-373.

Stotz, D.F.; Saboya, P.; Laverde-R, O. 2016. Aves. In: Pitman, N.; Bravo, A.; Claramunt, S.; Vriesendorp, C.; Alvira, D.; Ravikumar, A.; del Campo, A.; Stotz, D.F.; Wachter, T.; Heilpern, S.; Rodríguez, B.; Sáenz, A.R.; Smith R.C. 
(Eds). Perú: Medio Putumayo - Algodón. Rapid biological and social inventories, Report 28. The Field Museum, Chicago, Illinois. p. 131140; 466-492.

Systat Software Inc. 2008. SigmaPlot $®$ - Scientific data analysis and graphing software. San Jose, CA, US.

Vásquez-Arévalo, F.A.; Díaz Alván, J. 2019. Aves. In: Pérez-Peña, P.E.; Ramos-Rodríguez, M.C.; Díaz-Alván, J.; Zárate-Gómez, R.; Mejía, K. (Eds). Biodiversidad en la cuenca alta del Putumayo, Perú. Instituto de Investigaciones de la Amazonía Peruana. Iquitos, Perú. p. 106-131.

Xeno-Canto Foundation. 2019. Xeno-Canto: compartiendo cantos de aves de todo el mundo. (http://www.xeno-canto.org). Acceso 05/06/2019.

Yahuarcani, A.; Morote, K.; Calle, A.; Chujandama, M. 2009. Estado de conservación de Crax globulosa en la Reserva Nacional PacayaSamiria, Loreto. Revista Peruana de Biología, 15(2): 041-049. DOI: https://doi.org/10.15381/rpb. v15i2.1720

Zaccagnini, M.E.; Thompson, J.J.; Bernardos, J.; Calamari, N.; Goijman, A; Canavelli, S. 2011.
Riqueza, ocupación y roles funcionales potenciales de las aves en relación a los usos de la tierra y la productividad de los agroecosistemas: un ejemplo en la ecorregión pampeana. In: Laterra, P.; Jobbágy, E.G.; Paruelo, J.M. (Eds). Valoración de servicios ecosistémicos. Conceptos, herramientas, y aplicaciones para el ordenamiento territorial. Ediciones INTA, Buenos Aires. p. 185-219.

Zárate, R.; Cohello, G.; Palacios, J.J.; Escobedo, R.; Calvache, S; Vásquez, V.A. 2019a. Vegetación y flora. In: Pérez-Peña, P.E.; Ramos-Rodríguez, M.C.; Díaz, J.; Zárate R.; Mejía K. (Eds). Biodiversidad en la cuenca alta del Putumayo, Perú. Instituto de Investigaciones de la Amazonía Peruana. Iquitos, Perú. p. 15-56.

Zárate, R.; Mori, T.J.; Mozombite, L.F; Palacios, J.J.; Valles, L.A.; Cohello, G. 2019b. Vegetación y flora. In: Pérez-Peña, P.E.; Ramos-Rodríguez, M.C.; Díaz, J.; Zárate, R.; Mejía, K. (Eds). Biodiversidad en las cuencas del Napo y Curaray, Perú. Instituto de Investigaciones de la Amazonía Peruana; Centro para el Desarrollo del Indígena Amazónico. Iquitos, Perú. p. 1552. 
Apéndice 1: Lista de especies de aves en el interfluvio Napo-Putumayo-Amazonas. Donde: VU: Vulnerable, EN: En peligro, CR: En peligro crítico. NB: Especies migratorias que ocurren regularmente en Perú, pero solo en su período no reproductivo; V: Especies (errantes) que ocurren ocasionalmente en Perú y no son parte de la avifauna habitual,

H: Especies hipotéticas cuyos registros están basados solamente en observaciones, especímenes de dudosa procedencia, fotografías no publicadas o grabaciones mantenidas en manos privadas.

\begin{tabular}{|c|c|c|c|c|c|}
\hline Orden & Familia & Especie & MINAGRI & IUCN & CITES \\
\hline \multirow[t]{8}{*}{ Tinamiformes } & Tinamidae & Tinamus major & & & \\
\hline & & Tinamus guttatus & & & \\
\hline & & Crypturellus cinereus & & & \\
\hline & & Crypturellus soui & & & \\
\hline & & Crypturellus undulatus & & & \\
\hline & & Crypturellus duidae & VU & & \\
\hline & & Crypturellus variegatus & & & \\
\hline & & Crypturellus bartletti & & & \\
\hline \multirow[t]{3}{*}{ Anseriformes } & Anhimidae & Anhima cornuta & & & \\
\hline & Anatidae & Cairina moschata & & & \\
\hline & & Spatula discors (NB) & & & \\
\hline \multirow[t]{8}{*}{ Galliformes } & Cracidae & Penelope jacquacu & & & \\
\hline & & Pipile cumanensis & & & \\
\hline & & Ortalis guttata & & & \\
\hline & & Nothocrax urumutum & & & \\
\hline & & Crax globulosa & $\mathrm{CR}$ & EN & \\
\hline & & Mitu salvini & VU & & \\
\hline & & Mitu tuberosum & & & \\
\hline & Odontophoridae & Odontophorus gujanensis & & & \\
\hline \multirow[t]{9}{*}{ Columbiformes } & Columbidae & Columba livia & & & \\
\hline & & Patagioenas cayennensis & & & \\
\hline & & Patagioenas plumbea & & & \\
\hline & & Patagioenas subvinacea & & $\mathrm{VU}$ & \\
\hline & & Geotrygon montana & & & \\
\hline & & Leptotila verreauxi & & & \\
\hline & & Leptotila rufaxilla & & & \\
\hline & & Claravis pretiosa & & & \\
\hline & & Columbina talpacoti & & & \\
\hline \multirow[t]{4}{*}{ Cuculiformes } & Cuculidae & Crotophaga major & & & \\
\hline & & Crotophaga ani & & & \\
\hline & & Tapera naevia & & & \\
\hline & & Dromococcyx phasianellus & & & \\
\hline
\end{tabular}




\begin{tabular}{|c|c|c|c|c|c|}
\hline Orden & Familia & Especie & MINAGRI & IUCN & CITES \\
\hline & & Neomorphus pucheranii & & & \\
\hline & & Piaya cayana & & & \\
\hline & & Piaya melanogaster & & & \\
\hline & & Coccyzus americanus & & & \\
\hline \multirow[t]{5}{*}{ Nyctibiiformes } & Nyctibiidae & Nyctibius grandis & & & \\
\hline & & Nyctibius aethereus & & & \\
\hline & & Nyctibius griseus & & & \\
\hline & & Nyctibius leucopterus & VU & & \\
\hline & & Nyctibius bracteatus & & & \\
\hline \multirow[t]{8}{*}{ Caprimulgiformes } & Caprimulgidae & Chordeiles rupestris & & & \\
\hline & & Chordeiles minor (NB) & & & \\
\hline & & Lurocalis semitorquatus & & & \\
\hline & & Nyctiprogne leucopyga & & & \\
\hline & & Nyctidromus albicollis & & & \\
\hline & & Hydropsalis maculicaudus & & & \\
\hline & & Hydropsalis climacocerca & & & \\
\hline & & Nyctiphrynus ocellatus & & & \\
\hline \multirow[t]{18}{*}{ Apodiformes } & Apodidae & Streptoprocne zonaris & & & \\
\hline & & Chaetura cinereiventris & & & \\
\hline & & Chaetura egregia & & & \\
\hline & & Chaetura chapmani & & & \\
\hline & & Chaetura brachyura & & & \\
\hline & & Tachornis squamata & & & \\
\hline & & Panyptila cayennensis & & & \\
\hline & Trochilidae & Topaza pyra & & & II \\
\hline & & Florisuga mellivora & & & II \\
\hline & & Eutoxeres aquila & & & II \\
\hline & & Glaucis hirsutus & & & II \\
\hline & & Threnetes leucurus & & & II \\
\hline & & Phaethornis atrimentalis & & & II \\
\hline & & Phaethornis ruber & & & II \\
\hline & & Phaethornis hispidus & & & II \\
\hline & & Phaethornis bourcieri & & & II \\
\hline & & Phaethornis longirostris & & & II \\
\hline & & Phaethornis malaris & & & II \\
\hline
\end{tabular}




\begin{tabular}{|c|c|c|c|c|}
\hline Orden & Familia & Especie & MINAGRI & CITES \\
\hline & & Heliothryx auritus & & II \\
\hline & & Anthracothorax nigricollis & & II \\
\hline & & Heliodoxa schreibersii & & II \\
\hline & & Heliodoxa aurescens & & II \\
\hline & & Heliomaster longirostris & & II \\
\hline & & Chlorostilbon mellisugus & & II \\
\hline & & Campylopterus largipennis & & II \\
\hline & & Thalurania furcata & & II \\
\hline & & Chrysuronia oenone & & II \\
\hline & & Chionomesa fimbriata & & II \\
\hline & & Hylocharis sapphirina & & II \\
\hline Opisthocomiformes & Opisthocomidae & Opisthocomus hoazin & & \\
\hline \multirow[t]{8}{*}{ Gruiformes } & Psophiidae & Psophia crepitans & & \\
\hline & Rallidae & Porphyrio martinica & & \\
\hline & & Porphyrio flavirostris (NB) & & \\
\hline & & Laterallus melanophaius & & \\
\hline & & Mustelirallus albicollis & & \\
\hline & & Amaurolimnas concolor & & \\
\hline & & Aramides cajaneus & & \\
\hline & Heliornithidae & Heliornis fulica & & \\
\hline \multirow[t]{13}{*}{ Charadriiformes } & Charadriidae & Vanellus cayanus & & \\
\hline & & Vanellus chilensis & & \\
\hline & & Charadrius collaris & & \\
\hline & Scolopacidae & Gallinago paraguaiae & & \\
\hline & & Actitis macularius (NB) & & \\
\hline & & Tringa solitaria (NB) & & \\
\hline & & Tringa melanoleuca (NB) & & \\
\hline & & Tringa flavipes (NB) & & \\
\hline & Jacanidae & Jacana jacana & & \\
\hline & Rynchopidae & Rynchops niger & & \\
\hline & Laridae & Leucophaeus atricilla (NB) & & \\
\hline & & Sternula superciliaris & & \\
\hline & & Phaetusa simplex & & \\
\hline Eurypygiformes & Eurypygidae & Eurypyga helias & & \\
\hline \multirow[t]{2}{*}{ Suliformes } & Anhingidae & Anhinga anhinga & & \\
\hline & Phalacrocoracidae & Phalacrocorax brasilianus & & \\
\hline
\end{tabular}




\begin{tabular}{|c|c|c|c|c|c|}
\hline Orden & Familia & Especie & MINAGRI & IUCN & CITES \\
\hline \multirow[t]{17}{*}{ Pelecaniformes } & Ardeidae & Tigrisoma lineatum & & & \\
\hline & & Agamia agami & & VU & \\
\hline & & Cochlearius cochlearius & & & \\
\hline & & Zebrilus undulatus & & & \\
\hline & & Ixobrychus exilis & & & \\
\hline & & Nycticorax nycticorax & & & \\
\hline & & Butorides striata & & & \\
\hline & & Bubulcus ibis & & & \\
\hline & & Ardea cocoi & & & \\
\hline & & Ardea alba & & & \\
\hline & & Pilherodius pileatus & & & \\
\hline & & Egretta thula & & & \\
\hline & & Egretta caerulea & & & \\
\hline & Threskiornithidae & Eudocimus ruber (V) & & & \\
\hline & & Mesembrinibis cayennensis & & & \\
\hline & & Phimosus infuscatus (V) & & & \\
\hline & & Platalea ajaja & & & \\
\hline \multirow[t]{4}{*}{ Cathartiformes } & Cathartidae & Sarcoramphus papa & & & \\
\hline & & Coragyps atratus & & & \\
\hline & & Cathartes aura & & & \\
\hline & & Cathartes melambrotus & & & \\
\hline \multirow[t]{15}{*}{ Accipitriformes } & Pandionidae & Pandion haliaetus & & & II \\
\hline & Accipitridae & Gampsonyx swainsonii & & & II \\
\hline & & Chondrohierax uncinatus & & & II \\
\hline & & Leptodon cayanensis & & & II \\
\hline & & Elanoides forficatus & & & II \\
\hline & & Morphnus guianensis & & & II \\
\hline & & Harpia harpyja & VU & & 1 \\
\hline & & Spizaetus tyrannus & & & II \\
\hline & & Spizaetus ornatus & & & II \\
\hline & & Busarellus nigricollis & & & II \\
\hline & & Helicolestes hamatus & & & II \\
\hline & & Harpagus bidentatus & & & II \\
\hline & & Ictinia plumbea & & & II \\
\hline & & Accipiter superciliosus & & & II \\
\hline & & Accipiter bicolor & & & II \\
\hline
\end{tabular}




\begin{tabular}{|c|c|c|c|c|}
\hline Orden & Familia & Especie & MINAGRI & CITES \\
\hline & & Geranospiza caerulescens & & II \\
\hline & & Buteogallus schistaceus & & II \\
\hline & & Buteogallus urubitinga & & II \\
\hline & & Rupornis magnirostris & & II \\
\hline & & Pseudastur albicollis & & II \\
\hline & & Leucopternis melanops & & II \\
\hline & & Buteo nitidus & & II \\
\hline & & Buteo platypterus (NB) & & II \\
\hline & & Buteo brachyurus & & II \\
\hline \multirow[t]{8}{*}{ Strigiformes } & Strigidae & Megascops choliba & & II \\
\hline & & Megascops watsonii & & II \\
\hline & & Lophostrix cristata & & II \\
\hline & & Pulsatrix perspicillata & & II \\
\hline & & Ciccaba huhula & & II \\
\hline & & Ciccaba virgata & & II \\
\hline & & Glaucidium hardyi & & II \\
\hline & & Glaucidium brasilianum & & II \\
\hline \multirow[t]{7}{*}{ Trogoniformes } & Trogonidae & Pharomachrus pavoninus & & \\
\hline & & Trogon melanurus & & \\
\hline & & Trogon viridis & & \\
\hline & & Trogon ramonianus & & \\
\hline & & Trogon curucui & & \\
\hline & & Trogon rufus & & \\
\hline & & Trogon collaris & & \\
\hline \multirow[t]{8}{*}{ Coraciiformes } & Momotidae & Electron platyrhynchum & & \\
\hline & & Baryphthengus martii & & \\
\hline & & Momotus momota & & \\
\hline & Alcedinidae & Megaceryle torquata & & \\
\hline & & Chloroceryle amazona & & \\
\hline & & Chloroceryle aenea & & \\
\hline & & Chloroceryle americana & & \\
\hline & & Chloroceryle inda & & \\
\hline \multirow[t]{4}{*}{ Galbuliformes } & Galbulidae & Galbalcyrhynchus leucotis & & \\
\hline & & Galbula albirostris & & \\
\hline & & Galbula tombacea & & \\
\hline & & Galbula chalcothorax & & \\
\hline
\end{tabular}




\begin{tabular}{|c|c|c|c|c|c|}
\hline \multirow[t]{18}{*}{ Orden } & Familia & Especie & MINAGRI & IUCN & CITES \\
\hline & & Galbula dea & & & \\
\hline & & Jacamerops aureus & & & \\
\hline & Bucconidae & Notharchus hyperrhynchus & & & \\
\hline & & Notharchus ordii & & & \\
\hline & & Notharchus tectus & & & \\
\hline & & Bucco macrodactylus & & & \\
\hline & & Bucco tamatia & & & \\
\hline & & Bucco capensis & & & \\
\hline & & Malacoptila fusca & & & \\
\hline & & Malacoptila rufa & & & \\
\hline & & Micromonacha lanceolata & & & \\
\hline & & Nonnula rubecula & & & \\
\hline & & Nonnula brunnea & & & \\
\hline & & Monasa nigrifrons & & & \\
\hline & & Monasa morphoeus & & & \\
\hline & & Monasa flavirostris & & & \\
\hline & & Chelidoptera tenebrosa & & & \\
\hline \multirow[t]{19}{*}{ Piciformes } & Capitonidae & Capito aurovirens & & & \\
\hline & & Capito auratus & & & \\
\hline & & Eubucco richardsoni & & & \\
\hline & Ramphastidae & Ramphastos tucanus & & VU & II \\
\hline & & Ramphastos vitellinus & & VU & II \\
\hline & & Selenidera reinwardtii & & & \\
\hline & & Pteroglossus inscriptus & & & \\
\hline & & Pteroglossus castanotis & & & \\
\hline & & Pteroglossus pluricinctus & & & \\
\hline & & Pteroglossus azara & & & \\
\hline & Picidae & Picumnus lafresnayi & & & \\
\hline & & Picumnus rufiventris & & & \\
\hline & & Melanerpes cruentatus & & & \\
\hline & & Dryobates passerinus & & & \\
\hline & & Dryobates affinis & & & \\
\hline & & Campephilus rubricollis & & & \\
\hline & & Campephilus melanoleucos & & & \\
\hline & & Dryocopus lineatus & & & \\
\hline & & Celeus torquatus & & & \\
\hline
\end{tabular}




\begin{tabular}{|c|c|c|c|c|}
\hline Orden & Familia & Especie & MINAGRI & CITES \\
\hline & & Celeus grammicus & & \\
\hline & & Celeus flavus & & \\
\hline & & Celeus elegans & & \\
\hline & & Piculus flavigula & & \\
\hline & & Piculus chrysochloros & & \\
\hline & & Colaptes punctigula & & \\
\hline \multirow[t]{10}{*}{ Falconiformes } & Falconidae & Herpetotheres cachinnans & & II \\
\hline & & Micrastur ruficollis & & II \\
\hline & & Micrastur gilvicollis & & II \\
\hline & & Micrastur mirandollei & & II \\
\hline & & Micrastur semitorquatus & & II \\
\hline & & Micrastur buckleyi & & II \\
\hline & & Ibycter americanus & & II \\
\hline & & Daptrius ater & & II \\
\hline & & Milvago chimachima & & II \\
\hline & & Falco rufigularis & & II \\
\hline \multirow[t]{20}{*}{ Psittaciformes } & Psittacidae & Touit huetii & VU & II \\
\hline & & Touit purpuratus & & II \\
\hline & & Brotogeris sanctithomae & & II \\
\hline & & Brotogeris versicolurus & & II \\
\hline & & Brotogeris cyanoptera & & II \\
\hline & & Pyrilia barrabandi & & II \\
\hline & & Pionus menstruus & & II \\
\hline & & Graydidascalus brachyurus & & II \\
\hline & & Amazona festiva & & II \\
\hline & & Amazona ochrocephala & & II \\
\hline & & Amazona farinosa & & II \\
\hline & & Amazona amazonica & & II \\
\hline & & Forpus modestus & & II \\
\hline & & Forpus xanthopterygius & & II \\
\hline & & Pionites melanocephalus & & II \\
\hline & & Pyrrhura melanura & & II \\
\hline & & Aratinga weddellii & & II \\
\hline & & Orthopsittaca manilatus & & II \\
\hline & & Ara ararauna & & II \\
\hline & & Ara macao & & 1 \\
\hline
\end{tabular}




\begin{tabular}{|c|c|c|c|c|c|}
\hline Orden & Familia & Especie & MINAGRI & IUCN & CITES \\
\hline & & Ara chloropterus & & & II \\
\hline & & Ara severus & & & II \\
\hline & & Psittacara leucophthalmus & & & II \\
\hline \multirow[t]{33}{*}{ Passeriformes } & Thamnophilidae & Euchrepomis spodioptila $(\mathrm{H})$ & & & \\
\hline & & Cymbilaimus lineatus & & & \\
\hline & & Frederickena unduliger & & & \\
\hline & & Frederickena fulva & & & \\
\hline & & Taraba major & & & \\
\hline & & Thamnophilus doliatus & & & \\
\hline & & Thamnophilus schistaceus & & & \\
\hline & & Thamnophilus murinus & & & \\
\hline & & Thamnophilus aethiops & & & \\
\hline & & Thamnophilus amazonicus & & & \\
\hline & & Thamnophilus praecox $(\mathrm{H})$ & & & \\
\hline & & Megastictus margaritatus & & & \\
\hline & & Neoctantes niger & & & \\
\hline & & Thamnomanes ardesiacus & & & \\
\hline & & Thamnomanes caesius & & & \\
\hline & & Isleria hauxwelli & & & \\
\hline & & Pygiptila stellaris & & & \\
\hline & & Epinecrophylla haematonota & & & \\
\hline & & Epinecrophylla ornata & & & \\
\hline & & Epinecrophylla erythrura & & & \\
\hline & & Myrmotherula brachyura & & & \\
\hline & & Myrmotherula ignota & & & \\
\hline & & Myrmotherula multostriata & & & \\
\hline & & Myrmotherula axillaris & & & \\
\hline & & Myrmotherula sunensis & & & \\
\hline & & Myrmotherula longipennis & & & \\
\hline & & Myrmotherula menetriesii & & & \\
\hline & & Dichrozona cincta & & & \\
\hline & & Herpsilochmus dugandi & & & \\
\hline & & Herpsilochmus sp. & & & \\
\hline & & Microrhopias quixensis & & & \\
\hline & & Hypocnemis peruviana & & & \\
\hline & & Hypocnemis hypoxantha & & & \\
\hline
\end{tabular}




\begin{tabular}{|c|c|c|c|c|c|}
\hline Orden & Familia & Especie & MINAGRI & IUCN & CITES \\
\hline & & Cercomacroides serva & & & \\
\hline & & Cercomacra cinerascens & & & \\
\hline & & Myrmoborus lugubris & & VU & \\
\hline & & Myrmoborus myotherinus & & & \\
\hline & & Hypocnemoides melanopogon & & & \\
\hline & & Sclateria naevia & & & \\
\hline & & Percnostola rufifrons & & & \\
\hline & & Myrmelastes schistaceus & & & \\
\hline & & Myrmelastes hyperythrus & & & \\
\hline & & Myrmelastes leucostigma & & & \\
\hline & & Sciaphylax castanea & & & \\
\hline & & Akletos melanoceps & & & \\
\hline & & Hafferia fortis & & & \\
\hline & & Myrmophylax atrothorax & & & \\
\hline & & Myrmornis torquata & & & \\
\hline & & Pithys albifrons & & & \\
\hline & & Gymnopithys leucaspis & & & \\
\hline & & Oneillornis lunulatus & & & \\
\hline & & Rhegmatorhina melanosticta & & & \\
\hline & & Hylophylax naevius & & & \\
\hline & & Hylophylax punctulatus & & & \\
\hline & & Willisornis poecilinotus & & & \\
\hline & & Phlegopsis nigromaculata & & & \\
\hline & & Phlegopsis erythroptera & & & \\
\hline & Conopophagidae & Conopophaga aurita & & & \\
\hline & & Conopophaga peruviana & & & \\
\hline & Grallariidae & Grallaria varia & & & \\
\hline & & Grallaria dignissima & & & \\
\hline & & Hylopezus macularius & & & \\
\hline & & Myrmothera campanisona & & & \\
\hline & Rhinocryptidae & Liosceles thoracicus & & & \\
\hline & Formicariidae & Formicarius colma & & & \\
\hline & & Formicarius analis & & & \\
\hline & & Chamaeza campanisona & & & \\
\hline & & Chamaeza nobilis & & & \\
\hline & Furnariidae & Sclerurus obscurior & & & \\
\hline
\end{tabular}




\begin{tabular}{|c|c|c|c|c|c|}
\hline Orden & Familia & Especie & MINAGRI & IUCN & CITES \\
\hline & & Sclerurus rufigularis & & & \\
\hline & & Sclerurus caudacutus & & & \\
\hline & & Certhiasomus stictolaemus & & & \\
\hline & & Sittasomus griseicapillus & & & \\
\hline & & Deconychura longicauda & & & \\
\hline & & Dendrocincla merula & & & \\
\hline & & Dendrocincla fuliginosa & & & \\
\hline & & Glyphorynchus spirurus & & & \\
\hline & & Dendrexetastes rufigula & & & \\
\hline & & Nasica longirostris & & & \\
\hline & & Dendrocolaptes certhia & & & \\
\hline & & Dendrocolaptes picumnus & & & \\
\hline & & Xiphocolaptes promeropirhynchus & & & \\
\hline & & Xiphorhynchus obsoletus & & & \\
\hline & & Xiphorhynchus ocellatus & & & \\
\hline & & Xiphorhynchus elegans & & & \\
\hline & & Xiphorhynchus guttatus & & & \\
\hline & & Dendroplex picus & & & \\
\hline & & Campylorhamphus trochilirostris & & & \\
\hline & & Campylorhamphus procurvoides & & & \\
\hline & & Lepidocolaptes duidae & & & \\
\hline & & Xenops tenuirostris & & & \\
\hline & & Xenops minutus & & & \\
\hline & & Berlepschia rikeri & & & \\
\hline & & Microxenops milleri & & & \\
\hline & & Anabazenops dorsalis & & & \\
\hline & & Philydor erythrocercum & & & \\
\hline & & Philydor pyrrhodes & & & \\
\hline & & Anabacerthia ruficaudata & & & \\
\hline & & Ancistrops strigilatus & & & \\
\hline & & Dendroma erythroptera & & & \\
\hline & & Clibanornis rubiginosus & & & \\
\hline & & Automolus rufipileatus & & & \\
\hline & & Automolus ochrolaemus & & & \\
\hline & & Automolus subulatus & & & \\
\hline & & Automolus infuscatus & & & \\
\hline
\end{tabular}




\begin{tabular}{|c|c|c|c|c|c|}
\hline Orden & Familia & Especie & MINAGRI & IUCN & CITES \\
\hline & & Thripophaga fusciceps & & & \\
\hline & & Cranioleuca gutturata & & & \\
\hline & & Mazaria propinqua & & & \\
\hline & & Synallaxis gujanensis & & & \\
\hline & & Synallaxis albigularis & & & \\
\hline & & Synallaxis rutilans & & & \\
\hline & Pipridae & Tyranneutes stolzmanni & & & \\
\hline & & Chiroxiphia pareola & & & \\
\hline & & Cryptopipo holochlora & & & \\
\hline & & Lepidothrix coronata & & & \\
\hline & & Heterocercus aurantiivertex & & & \\
\hline & & Manacus manacus & & & \\
\hline & & Pipra filicauda & & & \\
\hline & & Machaeropterus striolatus & & & \\
\hline & & Pseudopipra pipra & & & \\
\hline & & Ceratopipra erythrocephala & & & \\
\hline & Cotingidae & Phoenicircus nigricollis & & & \\
\hline & & Querula purpurata & & & \\
\hline & & Cephalopterus ornatus & & & \\
\hline & & Cotinga maynana & & & \\
\hline & & Cotinga cayana & & & \\
\hline & & Lipaugus vociferans & & & \\
\hline & & Gymnoderus foetidus & & & \\
\hline & Tityridae & Tityra inquisitor & & & \\
\hline & & Tityra cayana & & & \\
\hline & & Tityra semifasciata & & & \\
\hline & & Schiffornis major & & & \\
\hline & & Schiffornis turdina & & & \\
\hline & & Laniocera hypopyrra & & & \\
\hline & & lodopleura isabellae & & & \\
\hline & & Pachyramphus castaneus & & & \\
\hline & & Pachyramphus polychopterus & & & \\
\hline & & Pachyramphus marginatus & & & \\
\hline & & Pachyramphus minor & & & \\
\hline & Onychorhynchidae & Onychorhynchus coronatus & & & \\
\hline & & Terenotriccus erythrurus & & & \\
\hline
\end{tabular}




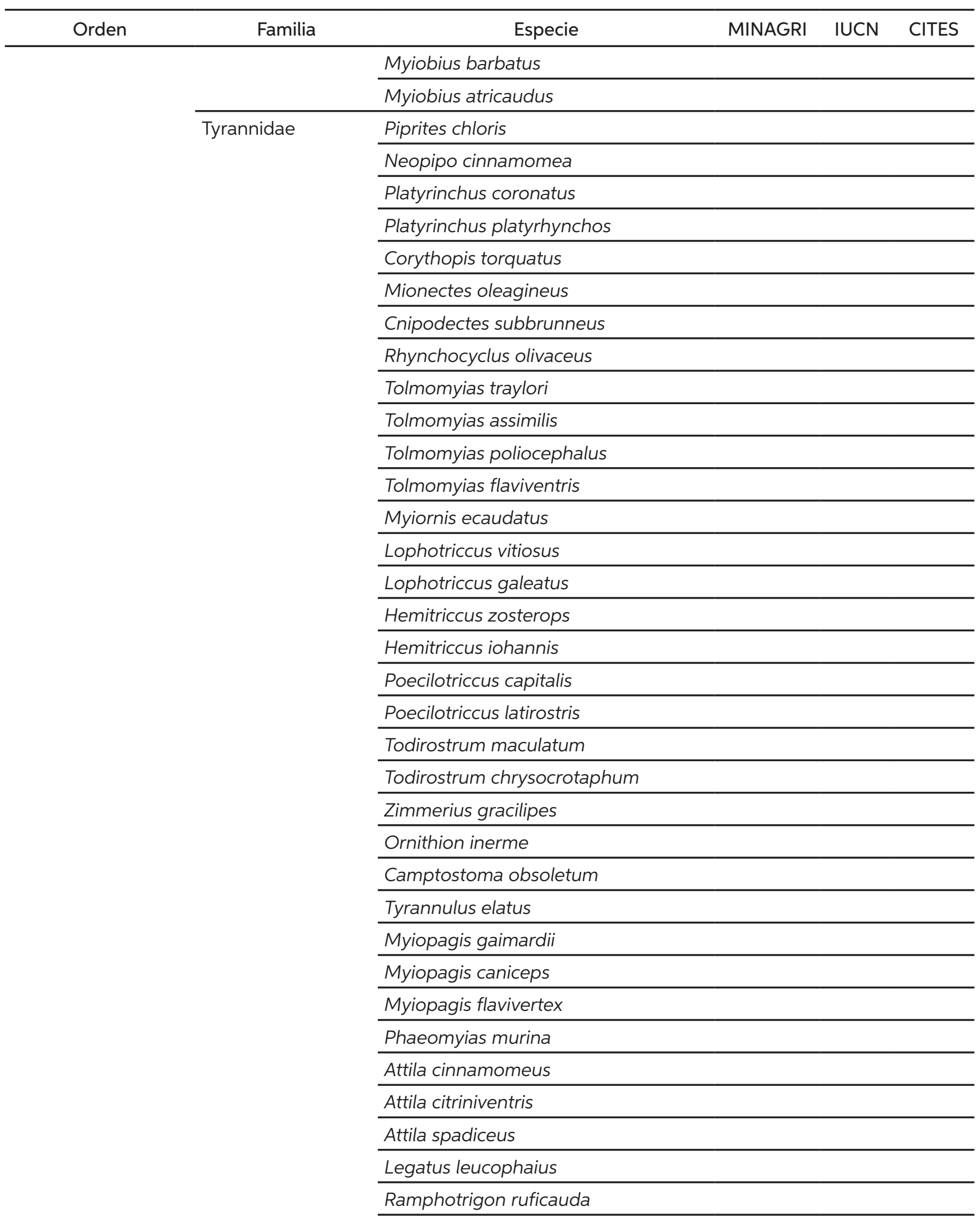




\begin{tabular}{|c|c|c|c|c|c|}
\hline Orden & Familia & Especie & MINAGRI & IUCN & CITES \\
\hline & & Pitangus sulphuratus & & & \\
\hline & & Pitangus lictor & & & \\
\hline & & Tyrannopsis sulphurea & & & \\
\hline & & Megarynchus pitangua & & & \\
\hline & & Myiodynastes luteiventris (NB) & & & \\
\hline & & Myiodynastes maculatus & & & \\
\hline & & Myiozetetes similis & & & \\
\hline & & Myiozetetes granadensis & & & \\
\hline & & Myiozetetes luteiventris & & & \\
\hline & & Conopias parvus & & & \\
\hline & & $\begin{array}{l}\text { Empidonomus aurantioatrocristatus } \\
\text { (NB) }\end{array}$ & & & \\
\hline & & Tyrannus melancholicus & & & \\
\hline & & Tyrannus savana (NB) & & & \\
\hline & & Tyrannus tyrannus (NB) & & & \\
\hline & & Rhytipterna simplex & & & \\
\hline & & Sirystes albocinereus & & & \\
\hline & & Myiarchus tuberculifer & & & \\
\hline & & Myiarchus ferox & & & \\
\hline & & Pyrocephalus rubinus & & & \\
\hline & & Knipolegus poecilocercus & & & \\
\hline & & Ochthornis littoralis & & & \\
\hline & & Lathrotriccus euleri & & & \\
\hline & & Empidonax alnorum & & & \\
\hline & & Contopus cooperi (NB) & & & \\
\hline & & Contopus virens (NB) & & & \\
\hline & Vireonidae & Hylophilus thoracicus & & & \\
\hline & & Vireolanius leucotis & & & \\
\hline & & Tunchiornis ochraceiceps & & & \\
\hline & & Pachysylvia hypoxantha & & & \\
\hline & & Vireo flavoviridis (NB) & & & \\
\hline & & Vireo olivaceus & & & \\
\hline & Corvidae & Cyanocorax violaceus & & & \\
\hline & Hirundinidae & Pygochelidon cyanoleuca & & & \\
\hline & & Atticora fasciata & & & \\
\hline & & Atticora tibialis & & & \\
\hline
\end{tabular}




\begin{tabular}{|c|c|c|c|c|c|}
\hline Orden & Familia & Especie & MINAGRI & IUCN & CITES \\
\hline & & \multicolumn{4}{|l|}{ Stelgidopteryx ruficollis } \\
\hline & & \multicolumn{4}{|l|}{ Progne tapera } \\
\hline & & \multicolumn{4}{|l|}{ Progne chalybea } \\
\hline & & \multicolumn{4}{|l|}{ Progne elegans (NB) } \\
\hline & & \multicolumn{4}{|l|}{ Tachycineta albiventer } \\
\hline & & \multicolumn{4}{|l|}{ Riparia riparia } \\
\hline & & \multicolumn{4}{|l|}{ Hirundo rustica } \\
\hline & & \multicolumn{4}{|l|}{ Petrochelidon pyrrhonota (NB) } \\
\hline & Troglodytidae & \multicolumn{4}{|l|}{ Microcerculus marginatus } \\
\hline & & \multicolumn{4}{|l|}{ Microcerculus bambla } \\
\hline & & \multicolumn{4}{|l|}{ Troglodytes aedon } \\
\hline & & \multicolumn{4}{|l|}{ Campylorhynchus turdinus } \\
\hline & & \multicolumn{4}{|l|}{ Pheugopedius coraya } \\
\hline & & \multicolumn{4}{|l|}{ Cantorchilus leucotis } \\
\hline & & \multicolumn{4}{|l|}{ Henicorhina leucosticta } \\
\hline & & \multicolumn{4}{|l|}{ Cyphorhinus arada } \\
\hline & Polioptilidae & \multicolumn{4}{|l|}{ Microbates collaris } \\
\hline & & \multicolumn{4}{|l|}{ Microbates cinereiventris } \\
\hline & & \multicolumn{4}{|l|}{ Ramphocaenus melanurus } \\
\hline & & \multicolumn{4}{|l|}{ Polioptila plumbea } \\
\hline & Donacobiidae & \multicolumn{4}{|l|}{ Donacobius atricapilla } \\
\hline & Turdidae & \multicolumn{4}{|l|}{ Catharus minimus (NB) } \\
\hline & & \multicolumn{4}{|l|}{ Catharus ustulatus (NB) } \\
\hline & & \multicolumn{4}{|l|}{ Turdus hauxwelli } \\
\hline & & \multicolumn{4}{|l|}{ Turdus lawrencii } \\
\hline & & \multicolumn{4}{|l|}{ Turdus ignobilis } \\
\hline & & \multicolumn{4}{|l|}{ Turdus albicollis } \\
\hline & Fringillidae & \multicolumn{4}{|l|}{ Euphonia chlorotica } \\
\hline & & Euphonia chrysopasta & & & \\
\hline & & Euphonia minuta & & & \\
\hline & & Euphonia laniirostris & & & \\
\hline & & Euphonia xanthogaster & & & \\
\hline & & Euphonia rufiventris & & & \\
\hline & Passerellidae & Ammodramus aurifrons & & & \\
\hline & Icteridae & Dolichonyx oryzivorus (NB) & & & \\
\hline & & Leistes militaris & & & \\
\hline
\end{tabular}




\begin{tabular}{|c|c|c|c|c|c|}
\hline Orden & Familia & Especie & MINAGRI & IUCN & CITES \\
\hline & & Psarocolius angustifrons & & & \\
\hline & & Psarocolius viridis & & & \\
\hline & & Psarocolius decumanus & & & \\
\hline & & Psarocolius bifasciatus & & & \\
\hline & & Cacicus solitarius & & & \\
\hline & & Cacicus cela & & & \\
\hline & & Cacicus latirostris & & & \\
\hline & & Cacicus haemorrhous & & & \\
\hline & & Cacicus oseryi & & & \\
\hline & & Icterus croconotus & & & \\
\hline & & Icterus cayanensis & & & \\
\hline & & Icterus chrysocephalus & & & \\
\hline & & Molothrus oryzivorus & & & \\
\hline & & Molothrus bonariensis & & & \\
\hline & & Lampropsar tanagrinus & & & \\
\hline & & Gymnomystax mexicanus & & & \\
\hline & Parulidae & Setophaga petechia (NB) & & & \\
\hline & & Myiothlypis fulvicauda & & & \\
\hline & & Cardellina canadensis (NB) & & & \\
\hline & Cardinalidae & Piranga rubra (NB) & & & \\
\hline & & Piranga olivacea (NB) & & & \\
\hline & & Habia rubica & & & \\
\hline & & Cyanoloxia cyanoides & & & \\
\hline & Thraupidae & Parkerthraustes humeralis & & & \\
\hline & & Chlorophanes spiza & & & \\
\hline & & Hemithraupis flavicollis & & & \\
\hline & & Volatinia jacarina & & & \\
\hline & & Loriotus cristatus & & & \\
\hline & & Loriotus luctuosus & & & \\
\hline & & Tachyphonus surinamus & & & \\
\hline & & Eucometis penicillata & & & \\
\hline & & Ramphocelus nigrogularis & & & \\
\hline & & Ramphocelus carbo & & & \\
\hline & & Lanio fulvus & & & \\
\hline & & Cyanerpes nitidus & & & \\
\hline & & Cyanerpes caeruleus & & & \\
\hline
\end{tabular}




\begin{tabular}{|c|c|c|c|c|c|}
\hline Orden & Familia & Especie & MINAGRI & IUCN & CITES \\
\hline & & Cyanerpes cyaneus & & & \\
\hline & & Tersina viridis & & & \\
\hline & & Dacnis albiventris & & & \\
\hline & & Dacnis lineata & & & \\
\hline & & Dacnis flaviventer & & & \\
\hline & & Dacnis cayana & & & \\
\hline & & Sporophila castaneiventris & & & \\
\hline & & Sporophila angolensis & & & \\
\hline & & Sporophila murallae & & & \\
\hline & & Saltator maximus & & & \\
\hline & & Saltator coerulescens & & & \\
\hline & & Saltator grossus & & & \\
\hline & & Paroaria gularis & & & \\
\hline & & Cissopis leverianus & & & \\
\hline & & Stilpnia nigrocincta & & & \\
\hline & & Tangara mexicana & & & \\
\hline & & Tangara chilensis & & & \\
\hline & & Tangara velia & & & \\
\hline & & Tangara callophrys & & & \\
\hline & & Tangara gyrola & & & \\
\hline & & Tangara schrankii & & & \\
\hline & & Thraupis episcopus & & & \\
\hline & & Thraupis palmarum & & & \\
\hline & & Ixothraupis xanthogastra & & & \\
\hline TOTAL & & 558 & 5 & 7 & 89 \\
\hline
\end{tabular}

Recibido: 14 de setiembre de 2020 Aceptado para publicación: 21 de octubre de 2020

Esta obra está bajo una Licencia Creative Commons Atribución-NoComercial-SinDerivar 4.0 Internacional. 